\title{
EFFICIENT AND ACCURATE KAM TORI CONSTRUCTION FOR THE DISSIPATIVE SPIN-ORBIT PROBLEM USING A MAP REDUCTION
}

\author{
RENATO CALLEJA, ALESSANDRA CELLETTI, JOAN GIMENO, AND RAFAEL DE LA LLAVE
}

\begin{abstract}
We consider the dissipative spin-orbit problem in Celestial Mechanics, which describes the rotational motion of a triaxial satellite moving on a Keplerian orbit subject to tidal forcing and drift.

Our goal is to construct quasi-periodic solutions with fixed frequency, satisfying appropriate conditions.

With the goal of applying rigorous KAM theory, we compute such quasi-periodic solution with very high precision. To this end, we have developed a very efficient algorithm. The first step is to compute very accurately the return map to a surface of section (using a high order Taylor's method with extended precision). Then, we find an invariant curve for the return map using recent algorithms that take advantage of the geometric features of the problem. This method is based on a rapidly convergent Newton's method which is guaranteed to converge if the initial error is small enough. So, it is very suitable for a continuation algorithm.

The resulting algorithm is quite efficient. We only need to deal with a one dimensional function. If this function is discretized in $N$ points, the algorithm requires $O(N \log N)$ operations and $O(N)$ storage. The most costly step (the numerical integration of the equation along a turn) is trivial to parallelize.

The main goal of the paper is to present the algorithms, implementation details and several sample results of runs.

We also present both a rigorous and a numerical comparison of the results of averaged and not averaged models.
\end{abstract}

Date: 18th June 2021.

Key words and phrases. Spin-orbit problem | Dissipation | Conformally symplectic systems | Tidal torque | Invariant curves.

R.C. was partially supported by UNAM-DGAPA PAPIIT Project IN 101020. A.C. has been partially supported the MIUR Excellence Department Project awarded to the Department of Mathematics, University of Rome Tor Vergata, CUP E83C18000100006, EU H2020 MSCA ETN StardustReloaded Grant Agreement 813644, and MIUR-PRIN 20178CJA2B "New Frontiers of Celestial Mechanics: theory and Applications". J.G. has been supported by the Spanish grants PGC2018-100699-B-I00 (MCIU/AEI/FEDER, UE), the Catalan grant 2017 SGR 1374 and MIUR-PRIN 20178CJA2B "New Frontiers of Celestial Mechanics: theory and Applications". The project leading to this application has also received funding from the European Union's Horizon 2020 research and innovation programme under the Marie Skłodowska-Curie grant agreement No 734557. J.G. thanks the School of Mathematics of GT for its hospitality in Spring 2019 and Fall 2019. R.L has been supported by NSF grant DMS 1800241. 


\section{$\S 1$. INTRODUCTION}

The construction of invariant structures in Celestial Mechanics and Astrodynamics has become of great importance in recent times, both for theoretical reasons and for the practical design of space missions. At present, many space missions are based on the determination of periodic and quasi-periodic orbits. Some notable examples of periodic/quasiperiodic orbits used in mission design are Lyapunov, Lissajous and halo orbits (see, e.g., CPS15, GM01, JM99]).

The existence and persistence of quasi-periodic orbits was developed by KAM theory (【Kol54, Arn63, Mos62]). Making KAM theory into a practical tool is an ongoing and rapidly progressing area, which applies to models of increasing complexity; it also leads to applications and has uncovered new mathematical phenomena.

With these motivations, this work develops a method for the construction of invariant tori in a concrete model of interest in Celestial Mechanics, namely the dissipative spinorbit problem (see Section $\S 2$, which describes the rotation about its center of an oblate moon orbiting a planet and subject to tidal forces. Our goal is to develop a method to compute quasi-periodic solutions in the spin-orbit problem. As it is well known, quasiperiodic orbits can be described geometrically as invariant tori on which the motion is conjugate to a rigid rotation. Hence, we will use indistinctly the names quasi-periodic solution and invariant (rotational) torus.

$\S 1.1$. Overview of the method. The method we develop starts by constructing a surface of section and a return map to it. The invariant tori for the flow correspond to invariant tori for the return map. We show that these return maps for the spin-orbit have the remarkable property that they transform the symplectic form into a multiple of itself. These maps are called conformally symplectic systems and enjoy several remarkable properties that lie at the root of a KAM theory and efficient algorithms (see Section $\$ 4.1$ ).

To find the invariant torus of the map, we follow the approach in CCdlL13b] and formulate a functional equation for the drift parameter and for the embedding of the torus, whose solutions are obtained formulating a quasi-Newton method that, given an approximate solution of the functional equation, produces another one with a quadratically small reminder. The quasi-Newton method in [CCdlL13b] takes advantage of the conformally symplectic geometric property.

The results of [CCdlL13b] guarantee that the method converges (as a double exponential, as Newton's methods) if the initial error is small enough (compared to some readily computable condition numbers).

The theorem in CCdlL13b] also shows that the difference between the true solution and the initial approximation is controlled by the error of the invariance equation, see eq. (26). Results of this form are called a-posteriori theorems in numerical analysis. We also note that one of the consequences of the work in CCdlL13b] is a local uniqueness for the solutions of (26) , except for composition for a rotation. This lack of uniqueness comes from the freedom on the choice of coordinates in the parameterization, but the geometric object and the drift parameter are locally unique.

Since the iterative method converges for small enough error, it will be used as the basis of a continuation method in parameters, which is guaranteed to converge until the 
assumptions in the theorem fail. Indeed, arguments in CdlL10 show that the method can be used as a practical way to compute the breakdown of the torus (see CC10] for an implementation to conformally symplectic maps). Note that the method is backed up by theorems and guaranteed to reach to the boundary of validity of the theorem, if given enough computational resources.

In this paper we will implement the method with extended arithmetic precision, motivated by the fact that the size of the error needed to apply the a-posteriori theorem is typically smaller than what can be obtained in double precision. With modern programming techniques, writing extended precision programs is not much more time consuming than using standard arithmetic. Of course, there is a penalty in speed, but since the algorithm is so efficient, one can still run comfortably even with extended precisions in today's desktop machines (see Section \$5.5 for details on timings and resources). Of course, in continuations, it is also possible to run the first iterations in double precision till the error is dominated by the double precision round-off and then run the final iterations in extended precision.

We have also used jet transport in order to get automatically the (first order) variational flow with respect to initial conditions and parameters. In [CCGdlL20b] we use the jet transport to get high order variational flows following the results in [GJJC 21 .

Finally, we have taken advantage of some modern advances such as multicore machines and multithreading given, for instance, continuation iterations in around $1 \mathrm{~min}$ when the initial guess is small enough. We did not explore other advanced architectures such as GPU, whose application in Celestial Mechanics is an interesting challenge. Some work on a simpler problem is in KAdlL21b.

$\S 1.2$. Efficiency and accuracy of the method. The use of return maps is very economical and natural. In the study of invariant tori for differential equations, it is standard to separate the directions along the flow and the transversal directions.

On the one hand, the torus remains very smooth along the directions of the flow for all values of the perturbation parameter. The flow in these directions can just be reduced to the well studied problem of computing solutions of ODE's and, in fact, there are many different algorithms suitable in different conditions.

On the other hand, the computation of the torus in the directions of the section is a much more complicated problem, since it requires KAM theory and the tori along these directions are much less regular; indeed, for large enough values of the perturbation, the tori may disappear. Nevertheless, even for values of the perturbation parameter where the tori do not exist, the solutions of the differential equations can be comfortably computed.

By dividing the problem into the KAM part for maps and the propagation to the return section, we reduce significantly the difficulty of the KAM part, since the tori have lower dimension. The computation of the return map is more complicated, but it is easily parallelizable and there are many studies on optimizing it. Hence the break up ends being rather advantageous. As indicated above, the methodologies of the two parts are very different and each of them can be fine tuned separately. 
The KAM part for maps is well documented in CCdlL13b; the algorithm therein applies quasi-Newton corrections and takes advantage of several identities related to the fact that the map is conformally symplectic.

The number of explicit steps of the KAM iterative procedure is about a dozen, see Algorithm 5.4. All the elementary steps are well structured vector operations that are primitives in modern languages or libraries, so that they are not too cumbersome to program.

Quite remarkably, all the steps are diagonal either in a grid representation of the function or in a Fourier representation. Of course, we can switch from one representation to the other using FFT. Hence, for a function discretized in $N$ modes, the quadratically convergent method requires only $O(N)$ storage and $O(N \log N)$ operations. Note that, in modern computers, the vector operations and the FFT are highly optimized, with specialized hardware.

These KAM algorithms have been implemented for maps given by explicit simple formulas [CC10, CF12, CCdlL20]. In theory, the only thing that one would need to do is to use the return map of the ODE (and its variational equations) in place of the explicit formulas. However, as we report in CCGdlL20a, in contrast with the explicit formulas that have few important harmonics, the return maps have many more relevant harmonics; this requires some adaptations and the phenomena observed are different.

§1.2.1. Relation with other methods. The methods of computing invariant tori based on normal form theory require working with functions with as many variables as the phase space, see [SL12, SL15]. In contrast, our methods require to manipulate only functions with as many variables as the dimension of the tori of the map. Reducing the number of variables in the unknown function is very important, since the number of operations needed to manipulate a function grows exponentially (with a large exponent) with the number of variables. Some recent papers that are also using return maps in Celestial Mechanics are, for instance, [HM21] (full dimensional tori in Hamiltonian systems) and KAdlL21a (whiskered tori, their stable and unstable manifolds and their intersections in Hamiltonian systems).

It is interesting to compare the methods developed here to Oli16, which uses a discretization of the tori without separating the tori and the flow directions. If the torus is discretized in $N$ points, this method requires $O\left(N^{2}\right)$ storage and $O\left(N^{3}\right)$ operations. Notice that $N$ points in 2-D tori give more or less the same precision as $N^{\frac{1}{2}}$ in 1-D tori.

A curious remark is that the linearization of the invariance equation (26) has a spectrum lying in circles as shown by [Mat68] (see AKdlL07, HdlL] for numerical experimentations), so that the Arnold-Kyrlov methods, successful in other models ([SNS10]), do not work very well. The method we use can be understdood as saying that, using geometric identities, we get the linearized equations to become constants (this phenomenon is called automatic reducibility).

One improvement in continuation methods, after an expensive effort in one step, is that one can compute inverses ([Mos73, Hal75]) or diagonalizations ([HdlL06, JO09]), perturbatively. These methods require still to store $O\left(N^{2}\right)$ storage and the perturbative calculations still require $O\left(N^{3}\right)$ operations even if the constants improve. 
$\S 1.3$. The model. We have implemented our results to the so-called dissipative spinorbit model ([Cel10]). In this section, we will review the physical bases of the model as well as formulate several variants (so-called, time-dependent friction (8) and averaged friction (9) ). These models will be analyzed (numerically and rigorously) in subsquent sections.

The spin-orbit model describes the rotational motion of a triaxial non-rigid satellite whose center of mass moves along an elliptic Keplerian orbit around a central planet. The spin-axis of the satellite is assumed to be perpendicular to the orbital plane and coinciding with the shortest physical axis. The rotation angle of the satellite is the angle between the longest axis of the satellite and a fixed direction, e.g. the periapsis line.

The motion of the rotation angle satisfies a second order differential equation depending periodically on time, through the orbital elements describing the osculating position of the center of mass of the satellite; such equation depends on two parameters, namely the orbital eccentricity and the equatorial flattening of the satellite. The model equations include a dissipative term due to the non-rigidity of the satellite, since the rotation gives rise to tides that dissipate energy and generate a torque. We adopt the model of [Pea05] in which the tidal torque is proportional to the angular velocity with a time-periodic coefficient. The dissipative term depends on two parameters: the orbital eccentricity and the dissipative factor, which is determined by the physical features of the satellite.

For typical bodies of the solar system, e.g. the Moon and many others among the biggest satellites, the force induced by the dissipation is much smaller than the conservative part, so that dissipation can be ignored in the description over short times. Nevertheless, since the dissipative forces have consequences that accumulate over time, they are very important in the description of long-term effects.

In the applied literature, it is very common to use a simplified version of the tidal torque that can be obtained by averaging the dissipation over time ([CC09, CL14, CL04]), so that the tidal torque becomes proportional to the derivative of the rotation angle.

One of the advantages of the method in this paper is that it provides a rather general rigorous justification of the averaging method for quasi-periodic solutions The basic idea is very simple: using standard averaging methods, we control the $2 \pi$ time map and, then, the effect of changing the map is controlled by the a-posteriori theorem (see Appendix (A). For the spin-orbit problem we also provide another justification that applies to all solutions.

Our formalism provides also rigorous estimates on the validity of the averaging approximation. Standard averaging theory can give estimates on the difference between the return maps in the averaged model and the true model (notice that the time of flights in return maps is about 1 , so that controlling the averaging method to order 1 is very standard). Then, we can use the a-posteriori format of the KAM theory in CCdlL13b to obtain estimates on the difference between the KAM tori and the drift parameters in the averaged and non-averaged cases. This result may look surprising, since one obtains control on solutions (and drift) for very long times. Besides this very general perturbative argument, in Appendix A we present some elementary arguments that, taking advantage of the structure of the system, obtain non-perturbative results. 
We conclude by mentioning that the current work has several consequences: in CCGdlL20b] we study the quantitative verification of a-posteriori theorems and the quantitative condition numbers, while in CCGdlL20a] we explore numerically the boundary of validity of KAM theory and uncover several phenomena that deserve further mathematical investigation. We hope that this paper (and the companions CCGdlL20b and CCGdlL20a) can stimulate further research, for example turning the estimates in [CCGdlL20b] into rigorous computer assisted proofs, studying higher dimensional models, incorporating more advanced computer architectures and explaining the phenomena at breakdown.

$\S 1.4$. Organization of this paper. This work is organized as follows. In Section $\S 2$ we present the spin-orbit model with tidal torque. The numerical formulation of the spinorbit problem is given in Section \$3, while the spin-orbit map is derived in Section $\$ 4$. The algorithm for the construction of invariant attractors and its applications is presented in Section $\$ 5$. Finally, some conclusions are given in Section $\$ 6$.

\section{§2. ThE SPIN-ORBIT PROBLEM WITH TIDAL TORQUE}

Consider the motion of a rigid body, say a satellite $\mathcal{S}$, with a triaxial structure, rotating around an internal spin-axis and, at the same time, orbiting under the gravitational influence of a point-mass perturber, say a planet $\mathcal{P}$. A simple model that describes the coupling between the rotation and the revolution of the satellite goes under the name of spin-orbit problem, which has been extensively studied in the literature in different contexts (see, e.g., [Bel01, Cel90b, Cel90a, CL04, WPM84]). This model is based on some assumptions that we are going to formulate as follows. Let $\mathcal{A}<\mathcal{B}<\mathcal{C}$ denote the principal moments of inertia of the satellite $\mathcal{S}$; then, we assume that:

H1. The satellite $\mathcal{S}$ moves on an elliptic Keplerian orbit with semimajor axis $a$ and eccentricity $e$, and with the planet $\mathcal{P}$ in one focus;

H2. The spin-axis of the satellite coincides with the smallest physical axis of the ellipsoid, namely the axis with associated moment of inertia $\mathcal{C}$;

H3. The spin-axis is assumed to be perpendicular to the orbital plane;

H4. The satellite $\mathcal{S}$ is affected by a tidal torque, since it is assumed to be non-rigid.

We adopt the units of measure of time such that the orbital period, say $T_{\text {orb }}$, is equal to $2 \pi$, which implies that the mean motion $n=2 \pi / T_{\text {orb }}$ is equal to one.

We define the equatorial ellipticity as the parameter $\varepsilon>0$ given by

$$
\varepsilon=\frac{3}{2} \frac{\mathcal{B}-\mathcal{A}}{\mathcal{C}},
$$

which is a measure of the oblateness of the satellite. When $\varepsilon=0$, then $\mathcal{A}=\mathcal{B}$ which

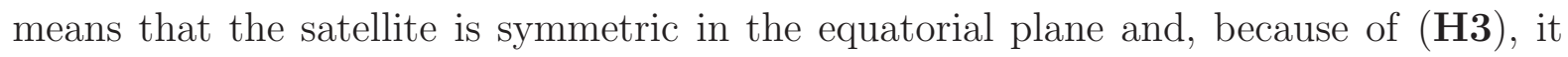
coincides with the orbital plane.

We consider the perturber $\mathcal{P}$ at the origin of an inertial reference frame with the horizontal axis coinciding with the direction of the semimajor axis. It is convenient to identify the orbital plane with the complex plane $\mathbb{C}$. The location of the center of 
mass of the rigid body $\mathcal{S}$ with respect to the perturber $\mathcal{P}$ is given, in exponential form, by $r \exp ($ i $f) \in \mathbb{C}$, where $r>0$ and $f$ are real functions depending on the time $t$ and they represent respectively the instantaneous orbital radius and the true anomaly of the Keplerian orbits. Indeed, over time, $r$ and $f$ describe an ellipse of eccentricity $e \in[0,1)$, semimajor axis $a$ and focus at the origin, see Figure 1.

Given that the mean motion $n$ has been normalized to one, then the mean anomaly coincides with the time $t$. By Kepler's equation ([Cel10]), we have the following relation between the eccentric anomaly $u$ and the time:

$$
t=u-e \sin u .
$$

The expressions which relate $r$ and $f$ with the eccentric anomaly (and hence with time through (2)) are given by

$$
\begin{aligned}
r & =a(1-e \cos u), \\
r \exp (\text { i } f) & =a\left(\cos u-e+\mathrm{i} \sqrt{1-e^{2}} \sin u\right) .
\end{aligned}
$$

Notice that we are assuming in (4) that for $t=0, f(0)=u(0)=0$, and consequently, $f(\pi)=u(\pi)=\pi$ when $t=\pi$. We also recall the following relations between the Keplerian elements, that will be useful in the following:

$$
\cos f=\frac{\cos u-e}{1-e \cos u} \quad \text { and } \quad \sin f=\frac{\sqrt{1-e^{2}} \sin u}{1-e \cos u} .
$$

As for the rotational motion, let $x$ be the angle formed by the direction of the largest physical axis, which belongs to the orbital plane, due to the assumptions ( $(\underline{\mathbf{H} 2})$ and ( with the horizontal (or semimajor) axis $a$.

If we neglect dissipative forces, the equation of motion which gives the dependence of $x$ on time is given by the following expression ([Cel10]) to which we refer as the conservative spin-orbit equation:

$$
\frac{d^{2} x(t)}{d t^{2}}+\varepsilon\left(\frac{a}{r(t)}\right)^{3} \sin (2 x(t)-2 f(t))=0,
$$

where $\varepsilon>0$ is given in (11), $r(t)=r(u(t) ; e)$ in (3),$f(t)=f(u(t) ; e)$ in (15), and where $u$ is related to $t$ through (2).

If we now assume that the satellite is not rigid, then we must consider a tidal torque, say $\mathcal{T}_{d}$, that acts on the satellite. According to Mac64, Pea05, we can write the tidal torque as a linear function of the velocity:

$$
\mathcal{T}_{d}\left(\frac{d x(t)}{d t}, t\right)=-\eta\left(\frac{a}{r(t)}\right)^{6}\left(\frac{d x(t)}{d t}-\frac{d f(t)}{d t}\right)
$$

where $\eta>0$ is named the dissipative constant. Since we are interested in astronomical applications, we specify that $\eta$ depends on the physical and orbital features of the body 


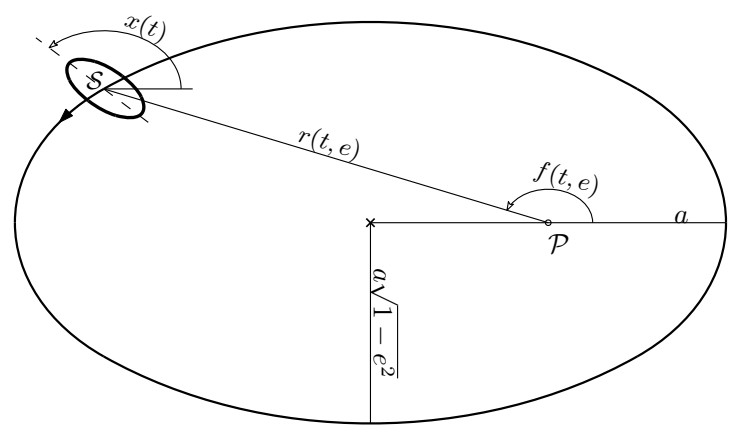

FiguRE 1. The spin-orbit problem: a triaxial satellite $\mathcal{S}$ moves around a planet $\mathcal{P}$ on an elliptic orbit with semimajor axis $a$ and eccentricity $e$. The position of the barycenter of $\mathcal{S}$ is given by the orbital radius $r$ and the true anomaly $f$. The rotational angle is denoted by $x$.

and takes the form

$$
\eta=3 n \frac{k_{2}}{\xi Q}\left(\frac{R_{e}}{a}\right)^{3} \frac{M}{m},
$$

where $k_{2}$ is the second degree potential Love number (depending on the structure of the body), $Q$ is the so-called quality factor (which compares the frequency of oscillation of the system to the rate of dissipation of energy), $\xi$ is a structure constant such that $C=\xi m R_{e}^{2}, R_{e}$ is the equatorial radius, $M$ is the mass of the central body $\mathcal{P}, m$ is the mass of the satellite $\mathcal{S}$. Astronomical observations suggest that for bodies like the Moon or Mercury the dissipative constant $\eta$ is of the order of $10^{-8}$.

The dynamics including the tidal torque is then described by the following equation to which we refer as the dissipative spin-orbit equation:

$$
\frac{d^{2} x(t)}{d t^{2}}+\varepsilon\left(\frac{a}{r(t)}\right)^{3} \sin (2 x(t)-2 f(t))=-\eta\left(\frac{a}{r(t)}\right)^{6}\left(\frac{d x(t)}{d t}-\frac{d f}{d t}\right) .
$$

The expression for the tidal torque can be simplified by assuming (as in [Pea05, CL04]) that the dynamics is essentially ruled by the average $\overline{\mathcal{T}}_{d}$ of the tidal torque over one orbital period, which can be written as

$$
\overline{\mathcal{T}}_{d}\left(\frac{d x}{d t}\right)=-\eta\left(\bar{L}(e) \frac{d x}{d t}-\bar{N}(e)\right)
$$

where (compare with $[$ Pea05])

$$
\begin{aligned}
\bar{L}(e) & \equiv \frac{1}{\left(1-e^{2}\right)^{9 / 2}}\left(1+3 e^{2}+\frac{3}{8} e^{4}\right), \\
\bar{N}(e) & \equiv \frac{1}{\left(1-e^{2}\right)^{6}}\left(1+\frac{15}{2} e^{2}+\frac{45}{8} e^{4}+\frac{5}{16} e^{6}\right) .
\end{aligned}
$$


When considering the averaged tidal torque, one is led to study the following equation of motion to which we refer as the averaged dissipative spin-orbit equation:

$$
\frac{d^{2} x(t)}{d t^{2}}+\varepsilon\left(\frac{a}{r(t)}\right)^{3} \sin (2 x(t)-2 f(t))=-\eta\left(\bar{L}(e) \frac{d x(t)}{d t}-\bar{N}(e)\right) .
$$

Note that in this model, we consider the average of the tidal forces, but do not average the conservative forces (see Appendix A). This is justified because, as indicated before, in practical problems, the dissipative forces are much smaller than the conservative ones.

Remarks 2.1. ( $\quad(i)$ The parameter $\varepsilon$ in (1) is zero only in the case of an equatorial symmetry with $\mathcal{A}=\mathcal{B}$. In that case, the equation of motion (6) is trivially integrable.

(ii) When $e=0$, the orbit is circular and therefore $r=a$ and $f=t+t_{0}$. Also in this case the equation of motion (6) is integrable.

(iii) The equation (6) is associated to the following one-dimensional, time-dependent Hamiltonian function:

$$
\mathcal{H}(y, x, t)=\frac{y^{2}}{2}-\frac{\varepsilon}{2}\left(\frac{a}{r(t)}\right)^{3} \cos (2 x-2 f(t)) .
$$

(iv) Equations (6), (8) and (10) are defined in a phase space which is a subset of $[0,2 \pi) \times \mathbb{R}$. Such a phase space can be endowed with the standard scalar product and a symplectic form $\Omega$, which in our case, is just the two dimensional area in phase space. Even if in two dimensional phase spaces the area (volume) is the same as the symplectic manifold, in systems with $N$ degrees of freedom $(N>1)$ the preservation of the two-form $\Omega$ is much more stringent than the preservation of the $2 N$ dimensional volume.

\section{§3. NUMERICAL VERSION OF THE SPIN-ORBIT PROBLEM}

To get a numerical representation of the ordinary differential equation (8) (equivalently (6) or (10)), it is convenient to express the equation in terms either of the eccentric anomaly $u$ or the mean anomaly which coincides with $t$. Although there is a clear bijection between $t$ and $u$ through (2), it seems reasonable to redefine everything in terms of the eccentric anomaly $u$ due to the expressions of $r$ in (3) and $f$ in (44). The procedure to get the equation of motion with $u$ as independent variable is the following.

The expression of $f$ in (5) is given easily in terms of $u$ (and $e$ ). Let $s(x)=s(x ; u, e)$ be the function defined as $s(x ; u, e):=\sin (2 x(t)-2 f(t))$, where the dependence on $u, e$ enters through $f$. Using trigonometric identities, we have an explicit expression in terms of $u$ and $e$ for the sinus in (8):

$$
s(x ; u, e)=\sin (2 x)\left(2 \cos ^{2} f-1\right)-\cos (2 x) 2 \cos f \sin f .
$$

Note here the useful relation for the derivatives of $s, c$ :

$$
\begin{aligned}
& \frac{\partial s}{\partial x}(x ; u, e)=2 c(x ; u, e), \\
& \frac{\partial c}{\partial x}(x ; u, e)=-2 s(x ; u, e),
\end{aligned}
$$


where

$$
c(x)=c(x ; u, e):=\cos (2 x)\left(2 \cos ^{2} f-1\right)+\sin (2 x) 2 \cos f \sin f .
$$

The time-change given by (2) leads to

$$
\frac{d f}{d t}=\left(\frac{a}{r}\right)^{2} \sqrt{1-e^{2}}
$$

As a consequence, equation (8) can be expressed in terms of the independent variable $u$ as

$$
\frac{d^{2} \beta(u)}{d u^{2}}-\frac{d \beta(u)}{d u} \frac{a}{r(u)} e \sin u+\varepsilon \frac{a}{r(u)} s(\beta)=-\eta\left(\frac{a}{r(u)}\right)^{5}\left(\frac{d \beta(u)}{d u}-\frac{a}{r(u)} \sqrt{1-e^{2}}\right)
$$

with $r$ defined in (3) and $s(\beta):=s(\beta ; u, e)$ given in $(12) 1$.

Note that the introduction of $u$ as independent variable implies a non-constant deformation in the angular component. More precisely, if $y(t)=\frac{d x(t)}{d t}$, defining

$$
\beta(u):=x(u-e \sin u),
$$

we obtain

$$
\gamma(u):=\frac{d \beta(u)}{d u}=\frac{r(u)}{a} y(u-e \sin u) .
$$

The ODE system (15) can now be integrated by any classical numerical integrator ([HNW93]) without having to solve the Kepler's equation (2) at each integration step. In the following sections, we will use a Taylor's integrator ([JZ05a]) that we briefly recall for self-consistency in Appendix B. We have used Taylor's method because it can produce solutions with high accuracy (say $10^{-30}$ ), since it can easily increase the orders of the Taylor's expansions in order to provide good enough trajectory values. These integrators are also used to produce rigorous enclosures ([BM98]). Both features seem to be important towards the goal of producing computer-assisted proofs (progress towards this goal will be reported in [CCGdlL20b]) or in the study of phenomena at breakdown that require delicate calculations not easy to make convincing (progress towards this goal will be reported in [CCGdlL20a]).

Even if not directly used in the present paper, we report in Appendix $\mathrm{C}$ the variational equations associated to (15). The variational equations with respect to coordinates and parameters can be used for different purposes (see [CCGdlL20b], CCGdlL20a]), e.g. to compute the parameterization of invariant structures, to get estimates based on the derivative of the flow, to compute chaos indicators like the Fast Lyapunov Indicator ([FLG97]).

\section{§4. THE CONFORMALLY SYMPLECTIC SPIN-ORBIT MAP}

In this section, we introduce the notion of conformally symplectic systems (Section \$4.1), we reduce the study of the spin-orbit problem to a discrete map (Section \$4.2) and we provide an explicit expression of the conformally symplectic factor (Section \$4.3).

\footnotetext{
${ }^{1}$ We abuse the notation referring $r(t)=a(1-e \cos u(t))$ and $r(u)=a(1-e \cos u$ ) as equal (similarly $f(t)$ and $f(u)$ ). Although formally we should label them differently, they are equivalent via the Kepler's equation.
} 
$\S 4.1$. Conformally symplectic systems. Conformally symplectic systems are dissipative systems that enjoy the remarkable property that they transform the symplectic form into a multiple of itself.

The formal definition for $2 n$-dimensional discrete and continuous systems is the following.

Let $\mathcal{M}=U \times \mathbb{T}^{n}$ be the phase space with $U \subseteq \mathbb{R}^{n}$ an open and simply connected domain with smooth boundary. We endow the phase space $\mathcal{M}$ with the standard scalar product and a symplectic form $\Omega$, represented by a matrix $J$ at the point $\underline{z}$ acting on vectors $\underline{u}, \underline{v} \in \mathbb{R}^{2 n}$ as $\Omega_{\underline{z}}(\underline{u}, \underline{v})=(\underline{u}, J(\underline{z}) \underline{v})$.

For the spin-orbit model, the symplectic form $\Omega$ is represented by the constant matrix $J$ which takes the form

$$
J=\left(\begin{array}{cc}
0 & 1 \\
-1 & 0
\end{array}\right) .
$$

Definition 4.1. A diffeomorphism $f$ on $\mathcal{M}$ is conformally symplectic if, and only if, there exists a function $\lambda: \mathcal{M} \rightarrow \mathbb{R}$ such that

$$
f^{*} \Omega=\lambda \Omega,
$$

where $f^{*}$ denotes the pull-back of $f$ (i.e., $f^{*} \Omega=\Omega \circ f$ ) and $\lambda$ is called the conformal factor.

In the following, we will consider a family $f_{\mu}: \mathcal{M} \rightarrow \mathcal{M}$ of mappings and we will call $\mu \in \mathbb{R}$ the drift parameter. Correspondingly, we will replace (18) by

$$
f_{\mu}^{*} \Omega=\lambda \Omega .
$$

We notice that for $\lambda=1$, we recover the symplectic case. Moreover, as remarked in CCdlL13b, for $n=1$ any diffeomorphism is conformally symplectic with a conformal factor that might depend on the coordinates; in particular, when $\Omega$ is the standard area, one has either $\lambda(x)=\left|\operatorname{det}\left(D f_{\mu}(x)\right)\right|$ or $\lambda(x)=-\left|\operatorname{det}\left(D f_{\mu}(x)\right)\right|$. When $n \geqslant 2$, it follows that $\lambda$ is a constant (see, e.g., [Ban02, CCdlL13b]).

Definition 4.1 extends to continuous systems by the use of the Lie derivative.

Definition 4.2. We say that a vector field $X$ is a conformally symplectic flow if, and only if, denoting by $L_{X}$ the Lie derivative, there exists a function $\mu: \mathbb{R}^{2 n} \rightarrow \mathbb{R}$ such that

$$
L_{X} \Omega=\mu \Omega
$$

Denoting by $\Phi_{t}$ the flow at time $t$ of the vector field $X$, we observe that (19) implies that

$$
\left(\Phi_{t}\right)^{*} \Omega=e^{\mu t} \Omega
$$

In our applications we will consider a family of vector fields $X_{\sigma}$ depending on a drift parameter $\sigma \in \mathbb{R}$ and we will replace (19) by

$$
L_{X_{\sigma}} \Omega=\mu \Omega \text {. }
$$


In our applications we will also have to consider time-dependent vector fields. A time dependent vector field $X(t)$ is conformally symplectic when $L_{X(t)} \Omega=\mu(t) \Omega$. It is not difficult to show that $\Phi_{a}^{b}$, the diffeomorphism which takes initial conditions at time $a$ to the position at time $b$ (hence $\Phi_{a}^{c}=\Phi_{b}^{c} \circ \Phi_{a}^{b}$ ), satisfies

$$
\left(\Phi_{a}^{b}\right)^{*} \Omega=e^{\int_{a}^{b} \mu(s) d s} \Omega .
$$

Note that (20) implies that if $X(t)$ is periodic of period $T$, the conformal factor of $\Phi_{a}^{a+T}$ is independent of $a$ and it is equal to the conformal factor of a vector field with a constant $\bar{\mu}=\frac{1}{T} \int_{a}^{a+T} \mu(s) d s$. This will be useful in the justification of averaging (see Appendix $\mathrm{A}$ ).

The spin-orbit models described by (8) and (10) are both conformally symplectic. Let us start to show that the averaged system (10) is conformally symplectic. We write (10) as the first order system

$$
\begin{aligned}
& \dot{x}=y \\
& \dot{y}=-\varepsilon\left(\frac{a}{r}\right)^{3} \sin (2 x-2 f)-\mu(\dot{x}-v),
\end{aligned}
$$

where $\mu=\eta \bar{L}(e)$ and $v=\frac{\bar{N}(e)}{L(e)}$. Hence, $\mu$ is the conformal factor and $v$ is the drift parameter. Denoting by $i_{X}$ the interior product and recalling that $\Omega=d y \wedge d x$, we have

$$
i_{X} \Omega=\dot{y} d x-\dot{x} d y=\left[-\mu(y-v)-\varepsilon\left(\frac{a}{r}\right)^{3} \sin (2 x-2 f)\right] d x-y d y .
$$

Then, we have

$$
d\left(i_{X} \Omega\right)=-\mu d y \wedge d x=-\mu \Omega
$$

Since

$$
L_{X} \Omega=i_{X} d \Omega+d\left(i_{X} \Omega\right)=d\left(i_{X} \Omega\right)
$$

we conclude that

$$
L_{X} \Omega=-\mu \Omega .
$$

A similar computation shows that the model described by (8) is conformally symplectic.

§4.2. The spin-orbit map. We introduce the Poincaré map associated to (8) or (10), which allows one to reduce the continuous spin-orbit problem to a discrete system. We denote by $G_{e}$ the flow at time $2 \pi$ in the independent variable $u$ associated to the equation of motion (15) in the coordinates $(\beta, \gamma)$ :

$$
G_{e}\left(\beta_{0}, \gamma_{0}\right)=\left(\begin{array}{l}
\beta\left(2 \pi ; \beta_{0}, \gamma_{0}, e\right) \\
\gamma\left(2 \pi ; \beta_{0}, \gamma_{0}, e\right)
\end{array}\right)
$$

with $\beta\left(2 \pi ; \beta_{0}, \gamma_{0}, e\right)$ and $\gamma\left(2 \pi ; \beta_{0}, \gamma_{0}, e\right)$ denoting the solution at $u=2 \pi$ with initial conditions $\left(\beta_{0}, \gamma_{0}\right)$ at $u=0$. If we set $G_{e}=\left(G_{e}^{(1)}, G_{e}^{(2)}\right)$, then the Poincaré map associated to (15) is

$$
\begin{aligned}
& \bar{\beta}=G_{e}^{(1)}(\beta, \gamma) \\
& \bar{\gamma}=G_{e}^{(2)}(\beta, \gamma) .
\end{aligned}
$$


The Poincaré map $P_{e}$ at time $t=2 \pi$ associated to (8) is then given by the conjugacy

$$
P_{e}=\Psi_{e}^{-1} \circ G_{e} \circ \Psi_{e}
$$

with the (time) change of coordinates from $(x, y) /(2 \pi)$ to $(\beta, \gamma)$ given by

$$
\Psi_{e}=2 \pi\left(\begin{array}{cc}
1 & 0 \\
0 & 1-e
\end{array}\right) .
$$

Using the map $G_{e}$ is very advantageous in numerical numerical implementation because this allows us to avoid to dealing with the Kepler's equation (2). On the other hand, the map $P_{e}$ is appropriate for physical interpretations, and the close and explicit relation among them (22) allows to choose the most advantageous one for the task at hand.

§4.3. The conformally symplectic factor. Our next task is to find an explicit form for the conformally symplectic factor $\lambda$ of the Poincaré map associated to (8) or equivalently (15).

By the Jacobi-Liouville Theorem, the determinant of the differential of the Poincaré map is obtained by integrating the trace of the Jacobian matrix associated to (8). Since the determinant and the trace of a matrix are invariant under a change of basis, their values are the same if we compute the system (15) via the Jacobian with elements $a_{i j}$ given by

$$
\begin{aligned}
& a_{11}=0, \quad a_{21}=-2 \varepsilon \frac{a}{r(u)} c(\beta ; u, e), \\
& a_{12}=1, \quad a_{22}=e \frac{a}{r(u)} \sin u-\eta\left(\frac{a}{r(u)}\right)^{5} .
\end{aligned}
$$

This leads to the following expression for the conformal factor:

$$
\lambda=\exp \left(\int_{0}^{2 \pi}\left(e \frac{a}{r(u)} \sin u-\eta\left(\frac{a}{r(u)}\right)^{5}\right) d u\right) .
$$

It is remarkable that the integral in (24) can be computed analytically in terms of $\eta$ and $e$. To this end, we need the following preliminary result.

Lemma 4.3. If $e \in[0,1)$ and $r=a(1-e \cos u)$, then

$$
\int_{0}^{2 \pi}\left(\frac{a}{r(u)}\right)^{5} d u=\pi \frac{3 e^{4}+24 e^{2}+8}{4\left(1-e^{2}\right)^{9 / 2}} .
$$

Proof. Since $e \in[0,1)$, then $\left(\frac{a}{r}\right)^{5}$ has no poles in the unit circle. By the change of variables $z=\exp (\mathrm{i} u)$ and a straightforward application of the Residue Theorem, we obtain:

$$
\int_{0}^{2 \pi}\left(\frac{a}{r(u)}\right)^{5} d u=2 \pi \sum_{\substack{w \text { sing. } \\|w|<1}} \operatorname{Res}\left(\frac{2^{5} z^{5}}{\left(2 z-e z^{2}-e\right)^{5}}, w\right),
$$

where Res denotes the residue of a holomorphic function. To compute the residue, we need to evaluate the poles $\alpha_{ \pm}$which are given by $\alpha_{ \pm}=e^{-1}\left(1 \pm \sqrt{1-e^{2}}\right)$. Since $\left|\alpha_{-}\right|<1$, 
then by an explicit computation, we get:

$$
\begin{aligned}
\int_{0}^{2 \pi}\left(\frac{a}{r(u)}\right)^{5} d u & =2 \pi \frac{1}{4 !} \lim _{z \rightarrow \alpha_{-}} \frac{d^{4}}{d z^{4}}\left(\frac{-2^{5} z^{4}}{e^{5}\left(z-\alpha_{+}\right)^{5}}\right) \\
& =2 \pi \lim _{z \rightarrow \alpha_{-}} \frac{32\left(\alpha_{+}^{4}+16 \alpha_{+}^{3} z+36 \alpha_{+}^{2} z^{2}+16 \alpha_{+} z^{3}+z^{4}\right)}{e^{5}\left(\alpha_{+}-z\right)^{9}} \\
& =\pi \frac{3 e^{4}+24 e^{2}+8}{4\left(1-e^{2}\right)^{9 / 2}} .
\end{aligned}
$$

The above result leads to the following Corollary, which gives an explicit form of the conformal factor of the spin-orbit model described by (8) .

Corollary 4.4. The conformally symplectic factor of the $2 \pi$-time map of the spin-orbit problem with tidal torque given by the system (8) has the following expression:

$$
\lambda=\exp \left(-\eta \pi \frac{3 e^{4}+24 e^{2}+8}{4\left(1-e^{2}\right)^{9 / 2}}\right) .
$$

Remarks 4.5. (i) Note that the result in Corollary 4.4 is also valid under the change of time given in (2). This means that the $2 \pi$-time map associated to the system (15) has the same symplectic factor, since the determinant is invariant under a change of basis.

(ii) The conformally symplectic factor $\lambda$ can be either contractive, expansive or neutral. The value $\lambda$ has a clear dynamical interpretation: at each $2 \pi$-interval of time, the values move $\lambda$ far away from unity. We remark that in this work we are interested to the contractive case, namely $\eta>0$.

\section{§5. THE COMPUTATION OF INVARIANT ATTRACTORS}

We consider the model described by equation (8) and we provide an algorithm (see Section \$5.1 for the construction of KAM invariant attractors. The algorithm relies on the fact that, starting from an initial approximate solution, one can construct a better approximate solution. A possible choice for the initial approximate solution is presented in Section $\$ 5.2$. A validation of the goodness of the solution is considered in Section \$5.3, which provides some accuracy tests. The construction of the invariant attractors through the implementation of the algorithm described in Section $\$ 5.1$ requires some technical procedures, precisely multiple precision arithmetic (see Appendix D) and parallel computing (see Section §5.5). Examples of the application of Algorithm 5.4 are given in Section $\$ 5.6$.

§5.1. An algorithm for constructing invariant attractors. Invariant attractors for the map $P_{e}$ defined in (22) associated to the dissipative spin-orbit equation (8) can be obtained by implementing an efficient algorithm based on Newton's method; this algorithm is also used to give a rigorous proof of invariant tori through KAM theorem, see CCGdlL20b, as well as to give accurate bounds on the breakdown threshold, see CCGdlL20a. 
To introduce the algorithm, we need to fix the frequency $\omega$, that we are going to choose sufficiently irrational, see Definition 5.1, and we need to introduce invariant KAM attractors, see Definition 5.2 .

Definition 5.1. The number $\omega \in \mathbb{R}$ is said Diophantine of class $\tau$ and constant $\nu$ for $\tau \geqslant 1, \nu>0$, and briefly denoted as $\omega \in \mathcal{D}(\nu, \tau)$, if the following inequality holds:

$$
|\omega k-q|^{-1} \leqslant \nu^{-1}|k|^{\tau}
$$

for $q \in \mathbb{Z}, k \in \mathbb{Z} \backslash\{0\}$.

We remark that the sets of Diophantine numbers as in Definition 5.1 is such that their union over $\nu>0$ has full Lebesgue measure in $\mathbb{R}$.

Next, we define as follows an invariant attractor with frequency $\omega$ satisfying (25).

Definition 5.2. Let $P_{e}: \mathcal{M} \rightarrow \mathcal{M}$ be a family of conformally symplectic maps defined on a symplectic manifold $\mathcal{M} \subset \mathbb{R} \times \mathbb{T}$ and depending on the drift parameter e. A KAM attractor with frequency $\omega$ is an invariant torus described by an embedding $K_{p}: \mathbb{T} \rightarrow \mathcal{M}$ and a drift parameter $e_{p}$, satisfying the following invariant equation for $\theta \in \mathbb{T}$ :

$$
P_{e_{p}} \circ K_{p}(\theta)=K_{p}(\theta+\omega) .
$$

We will often write (26) in the form

$$
P_{e_{p}} \circ K_{p}=K_{p} \circ T_{\omega},
$$

where $T_{\omega}$ denotes the shift function by $\omega$, i.e., $T_{\omega}(\theta)=\theta+\omega$.

The solutions of the invariance equation (26) is unique up to a shift. For all real $\alpha$, if $\hat{K}_{p}(\theta):=K_{p}(\theta+\alpha)$, then $\left(\hat{K}_{p}, e_{p}\right)$ is also a solution of (26). Note that all these solutions parameterize the same geometric object. In [CCdIL13b there is a simple argument showing that this is the only source of lack of local uniqueness.

Remark 5.3. It is easy to see - even in the integrable case - that to obtain an attractor with a specific frequency we need to adjust the drift parameter.

Hence, repeating the calculation several times, we obtain the drift as a function of the frequency. One can invert this - one-dimensional - function and obtain the frequency as a function of the drift, so that the two are mathematically equivalent, see Figure Q2.

For astronomers, the frequency is directly observed and it is natural to think of using the measurements of the frequency to obtain values of the drift.

Theoretical physicists may prefer that the values of the drift are known and that one predicts the frequency.

Both points of view are mathematically equivalent modulo inverting a 1-D function. In astronomy, since there is little a-priori information on the values of the elastic properties of the satellites, it seems more natural to study the drift as function of the frequency. On other physical applications, where the values of the model are known from the start, the other point of view may be preferable.

One small technical problem (that can be solved) is that the function is not defined for all values of the frequency. The theory only establishes for a set of large measure. 
Repeatedly, not all the values of the drift parameter lead to a system that has a rotational attractor.

The starting point of the iterative process in the spin-orbit problem is then $(K, e)$, an approximate solution of the invariance equation (26),

$$
P_{e} \circ K(\theta)-K(\theta+\omega)=E(\theta),
$$

where the "error" $E$ is thought of as small (making precise the notion of small will require the introduction of norms).

Algorithm 5.4 below takes the pair $(K, e)$ and produces another approximate solution $(\tilde{K}, \tilde{e})$, which satisfies (26) up to an error whose norm is quadratically smaller with respect to $E$ (again, making all this precise requires introducing norms).

We note that all the steps are rather explicit operations taking derivatives, shifting and performing alebraic operations. The most delicate steps are 8, 12, which involve solving cohomology equations and step 9 which involves solving a $2 \times 2$ linear equation. The assumption of invertibility of this explicit $2 \times 2$ matrix is a non-degeneracy assumption that takes the place of the classical twist condition.

The Algorithm 5.4 is based on that described in [CCdlL13b] and adapted for the spinorbit problem. Even if, for the sake of simplicity, we only present the algebraic operations in the recipe, in CCdlL13b there are geometric interpretations that motivate the steps.

Algorithm 5.4 (Newton's method for finding a torus in the spin-orbit problem).

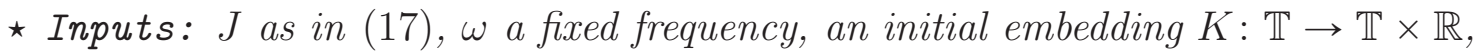
access to the $2 \pi$-time flow map $G_{e}$ of (15) for fixed values $\varepsilon$ and $\eta$, change of

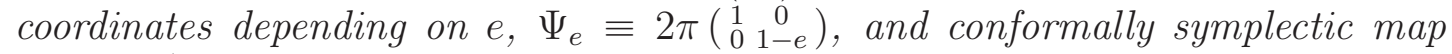
$P_{e} \equiv \Psi_{e}^{-1} \circ G_{e} \circ \Psi_{e}$.

* Output: New $K$ and e satisfying the invariance equation (26) up to a given tolerance.

$\star$ Notation: If $A$ is defined in $\mathbb{T}, \bar{A}:=\int_{\mathbb{T}} A$ and $A^{0}:=A-\bar{A}$.

1. $E \leftarrow P_{e} \circ K-K \circ T_{\omega}$, $E_{1} \leftarrow E_{1}-\operatorname{round}\left(E_{1}\right)$.

2. $\alpha \leftarrow D K$.

3. $N \leftarrow\left(\alpha^{t} \alpha\right)^{-1}$.

4. $M \leftarrow\left[\begin{array}{ll}\alpha & J^{-1} \alpha N\end{array}\right]$.

5. $\widetilde{E} \leftarrow\left(M^{-1} \circ T_{\omega}\right) E$.

6. $\lambda$ from the Corollary 4.4. 
7. $P \leftarrow \alpha N$,

$S \leftarrow\left(P \circ T_{\omega}\right)^{t} D P_{e} \circ K J^{-1} P$,

$\widetilde{A} \leftarrow M^{-1} \circ T_{\omega} D_{e} P_{e} \circ K$.

8. $\left(B_{a}\right)^{0}$ solving $\lambda\left(B_{a}\right)^{0}-\left(B_{a}\right)^{0} \circ T_{\omega}=-\left(\widetilde{E}_{2}\right)^{0}$, $\left(B_{b}\right)^{0}$ solving $\lambda\left(B_{b}\right)^{0}-\left(B_{b}\right)^{0} \circ T_{\omega}=-\left(\widetilde{A}_{2}\right)^{0}$.

9. Find $\bar{W}_{2}, \sigma$ solving the linear system

$$
\left(\begin{array}{cc}
\bar{S} & \overline{S\left(B_{b}\right)^{0}}+\overline{\widetilde{A}_{1}} \\
\lambda-1 & \overline{\widetilde{A}_{2}}
\end{array}\right)\left(\begin{array}{c}
\overline{W_{2}} \\
\sigma
\end{array}\right)=\left(\begin{array}{c}
-\overline{\widetilde{E}_{1}}-\overline{S\left(B_{a}\right)^{0}} \\
-\overline{\widetilde{E}_{2}}
\end{array}\right) .
$$

10. $\left(W_{2}\right)^{0} \leftarrow\left(B_{a}\right)^{0}+\sigma\left(B_{b}\right)^{0}$.

11. $W_{2} \leftarrow\left(W_{2}\right)^{0}+\bar{W}_{2}$.

12. $\left(W_{1}\right)^{0}$ solving $\left(W_{1}\right)^{0}-\left(W_{1}\right)^{0} \circ T_{\omega}=-\left(S W_{2}\right)^{0}-\left(\widetilde{E}_{1}\right)^{0}-\left(\widetilde{A}_{1}\right)^{0} \sigma$.

13. $K \leftarrow K+M W$, $e \leftarrow e+\sigma$.

Algorithm 5.4 needs some practical remarks:

- Because of the periodicity condition $K(\theta+1)=K(\theta)+\left(\begin{array}{l}1 \\ 0\end{array}\right)$, one can always define the periodic map $\tilde{K}(\theta):=K(\theta)-\left(\begin{array}{l}\theta \\ 0\end{array}\right)$ which, generically, admits a Fourier series. Then, one obtains that

$$
K \circ T_{\omega}=\tilde{K} \circ T_{\omega}+\left(\begin{array}{c}
\omega \\
0
\end{array}\right) .
$$

- The function $E$ in step 1 must perform the subtraction in the first component in $\mathbb{T}$. For a numerical implementation that can be fulfilled by the assignment $E_{1} \leftarrow$ $E_{1}-\operatorname{round}\left(E_{1}\right)$, where round returns the nearest integer value of its argument. Such a function is commonly provided in almost all programming languages.

- The matrix $M$ in step 4 is unimodular, which allows to get an easy inverse matrix expression.

- The quantities $D P_{e}$ and $D_{e} P_{e}$ in step 7 are needed to compute the directional variational flow of $\Phi$, which can be done automatically using the explanations in Section C.1.

- The stopping criterion is either that $\|E\|$ or $\max \{\|M W\|,|\sigma|\}$ is smaller than a prefixed tolerance.

A common numerical representation for periodic mappings is a Fourier series in the inputs $K_{1}$ and $K_{2}$, which gives us a representation of $K \equiv\left(K_{1}, K_{2}\right)$. In such a representation, we can use the Fourier transform, or its numerical version via Fast Fourier Transform (FFT) algorithm.

Therefore any periodic mapping, say $f$, admits two representations, namely in points (or table of values) and in Fourier coefficients. The first one is just the values $\left(\breve{f}_{k}\right)_{k=0}^{n-1}$ of the mapping in an equispaced mesh in $[0,1)$ of size $n$. The second one is obtained 
by the Inverse of the Fast Fourier Transform (IFFT), denoted by $\left(\widehat{f}_{k}\right)_{k=0}^{n-1}$. Notice that, because the function is assumed to be real-valued, the two representations can have the same size, i.e. $n$ real values.

Depending on the step in Algorithm 5.4, it may be better to use one representation or the other. For instance, $P_{e} \circ K$ in 1 , and $D P_{e} \circ K, D_{e} P_{e} \circ K$ in 7 are better when $K$ is in a table of values, although an ODE version of (8) in terms of Fourier coefficients can be considered.

On the other hand, the composition with $T_{\omega}$ and the solution of the cohomological equations in 8 and 12 are easier if $K$ is in Fourier series. Indeed, these equations can be solved in Fourier coefficients, using the following result whose proof is straightforward.

Lemma 5.5. Let $\eta(\theta)=\sum_{k} \eta_{k} \exp (2 \pi i k \cdot \theta)$ and let $\omega$ be irrational. Then:

(i) If $\eta_{0}=0$, then $\phi \circ T_{\omega}-\phi=\eta$ has solution $\phi(\theta)=\sum_{k} \phi_{k} \exp (2 \pi i k \cdot \theta)$ with

$$
\phi_{k}= \begin{cases}\frac{\eta_{k}}{\exp (2 \pi i k \cdot \omega)-1} & \text { if } k \neq 0, \\ 0 & \text { otherwise. }\end{cases}
$$

(ii) If $\lambda$ is not a root of unit, then $\phi \circ T_{\omega}-\lambda \phi=\eta$ has solution with coefficients

$$
\phi_{k}=\frac{\eta_{k}}{\exp (2 \pi i k \cdot \omega)-\lambda} .
$$

$\S 5.2$. Initial approximation of the invariant curve. Repeated application of the Newton's method from Algorithm 5.4 produces a very accurate solution provided that one can get a good enough initial approximation. In this section we address the problem of producing such an initial approximation. Although the methods are rather general, we are going to give the results for the cases of study in this paper. We select the following two frequencies with good Diophantine conditions belonging to the class $\mathcal{D}\left(\frac{2}{3-\sqrt{5}}, 1\right)$, see Definition 5.1 .

$$
\omega_{1}=\gamma_{g}^{+}
$$

and

$$
\omega_{2}=1+\frac{1}{2+\gamma_{g}^{-}},
$$

where we define $\gamma_{\frac{g}{g}}^{ \pm}=\frac{\sqrt{5} \pm 1}{2}$.

One method to provide an initial approximation is, of course, to continue from an integrable case that can be solved explicitly. Another method is to do an easy calculation of an approximate torus. Since the system is dissipative, the torus, if it exists, will be an attractor.

§5.2.1. A continuation method from the integrable case. Let us start to analyze the averaged problem for which we look for the drift starting from the integrable case. Fix the frequency $\omega$ and set $\varepsilon=0$ in (10); then, the drift $e$ can be chosen so that

$$
\frac{\bar{N}(e)}{\bar{L}(e)}=\omega \text {. }
$$


The above equation provides the eccentricity as a function of the frequency. In fact, as noticed in [CC09], for $\eta \neq 0$ the solution of (10) can be written as

$$
x(t)=x(0)+\frac{\bar{N}(e)}{\bar{L}(e)} t+\frac{1-\exp (-\eta t)}{\eta}\left(\dot{x}(0)-\frac{\bar{N}(e)}{\bar{L}(e)}\right),
$$

which shows that $\dot{x}=\frac{\bar{N}(e)}{L(e)}$ is a global attractor for the unperturbed, purely dissipative case $\varepsilon=0$. Setting $y=\dot{x}$, we can select as initial starting condition

$$
x(0)=0 \quad \text { and } \quad y(0)=\frac{\bar{N}(e)}{\bar{L}(e)} .
$$

We remark that, despite the use of the averaged version of the spin-orbit problem given in (10), to approximate an initial guess of the eccentricity in terms of the frequency, we can also use the non-averaged spin-orbit problem (8) to provide an approximated eccentricity, see Section $\$ 5.2 .2$.

§5.2.2. Direct iteration. We propose a method different from Section \$5.2.1, which takes advantage of the fact that the torus, if it exists, is an attractor. We do not need to consider the average equation and we can start from the model (8). Hence, we start by selecting a set of points at random; after a transient number of iterations (e.g., choose the transient as the inverse of the dissipation multiplied by a convenient safety factor), we expect that the orbit is close to the attractor. Then, we assess whether indeed this orbit has a rotation number.

Since not all the attractors of dissipative maps are rotational orbits, not all of them should have a rotation number. Of course, there may be situations where the orbit is a chaotic attractor that happens to have a rotation number.

Given the importance of the rotation number, there are quite a number algorithms to compute it, e.g. [LFC92, Ath98, Las99, ALM00, Las99, SV06, GMS10a, GMS10b, SNS10]. We have used the method in [DSSY17] which speeds the convergence to the rotation number. We will present more details of the calculation in CCGdlL20b]. We note that the method in [DSSY17] gives a very good indication of the existence of an invariant circle. In [DSSY17] it is shown that if there is a smooth invariant circle, the convergence of the method to a rotation number is very fast. Hence the fast convergence of the algorithm is a reasonably good evidence of the existence of a rotational torus. Of course, the convergence of the Newton's method started in this guess is a much stronger validation of the correctness of the guess.

Figure 2 compares the two approaches explained here and in Section $\$ 5.2 .1$. The first one is straightforward, since it consists in plotting the function $\bar{N}(e) / \bar{L}(e)$ in terms of the eccentricity $e$. The second one requires a little bit more effort, since it needs to numerically integrate (8) to compute the rotation number.

§5.2.3. Initial approximation for the embedding. Once we have fixed an initial guess for the eccentricity and an initial starting point, we can proceed to get an initial guess of the embedding $K$ which is needed as input in the Algorithm 5.4. To this end, we first perform a preliminary transient of iterations of $G_{e}$, defined in (21), at the initial point 


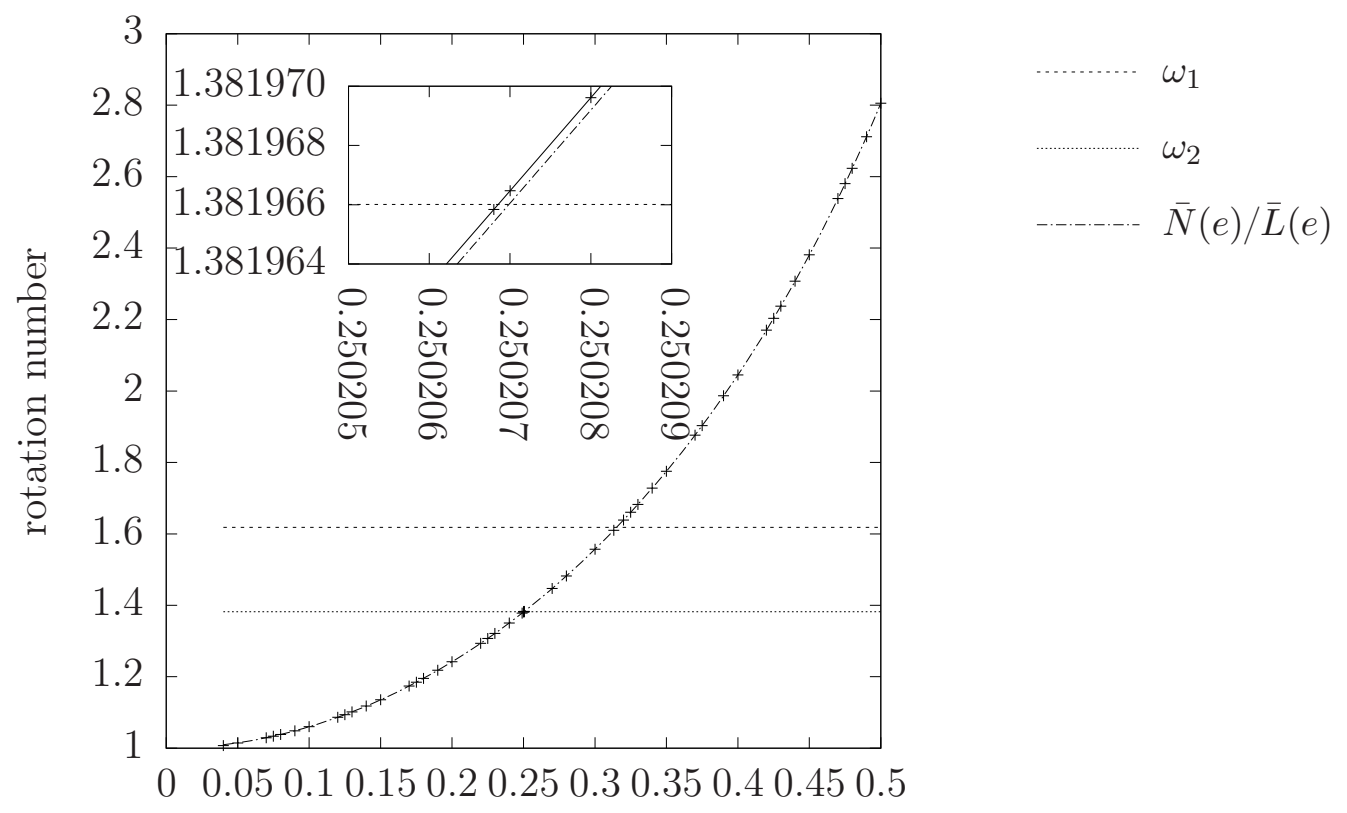

$e$

FIGURE 2. Eccentricity versus the rotation number denoted by + of the system (8) with $\varepsilon=10^{-4}$ and $\eta=10^{-5}$. Frequencies as in (28) and (29). The small window is just a zoom-in near to $\omega_{2}$ which shows that the averaged quantity $\bar{N}(e) / \bar{L}(e)$ approaches the non-averaged rotation number.

$\Psi_{e}(x(0), y(0) /(2 \pi))$, with $(x(0), y(0))$ given in (30). After that, we can follow the steps in Algorithm 5.6 to get the initial guess for $K$ for the Algorithm 5.4.

Algorithm 5.6 (Invariant curve approximation).

$\star$ Inputs: Points $\left(\beta_{k}, \gamma_{k}\right)_{k=0}^{n-1} \subset[0,2 \pi) \times \mathbb{R}$ from iteration of the $2 \pi$-time Poincaré map of (15) with parameter values $\varepsilon, \eta$, and $e$ and number of Lagrange interpolation points $2 j \in \mathbb{N}$.

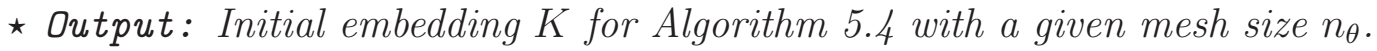

1. $\operatorname{Sort}\left(\beta_{i_{k}}, \gamma_{i_{k}}\right)_{k=0}^{n-1}$ such that $\beta_{i_{1}} \leqslant \cdots \leqslant \beta_{i_{n}}$.

2. Mesh $\bar{\beta}_{k}=2 \pi k / n_{\theta}$ with $k=0, \ldots, n_{\theta}-1$.

3. For each $k=0, \ldots, n_{\theta}-1$, let $\bar{\gamma}_{k}$ be the Lagrange interpolation centered at $\left(\beta_{i_{k}}, \gamma_{i_{k}}\right)$ with $2 j$ points $\gamma_{i_{k}-j}(\bmod n), \ldots, \gamma_{i_{k}+j}(\bmod n)$ and their respective abscissae.

4. Return the table of values $K \equiv\left(\Psi_{e}^{-1}\left(\bar{\beta}_{k}, \bar{\gamma}_{k}\right)\right)_{k=0}^{n_{\theta}-1}$ with $\Psi_{e}$ from (23).

$\S 5.3$. Accuracy tests. We are now looking for $K: \mathbb{T} \rightarrow \mathbb{T} \times \mathbb{R}$ and the parameter $e$ so that the invariance equation (26) is verified, for $P_{e}$ given in (22) and a fixed frequency $\omega$ like in (28) or (29). 
In this process, we have three main sources of error that affect the result.

E1. The error of the invariance condition on the table of values. This error is controlled by the Newton's procedure.

E2. The error on the integration. This error is controlled by the numerical integrator when we request absolute and relative tolerances.

E3. The error in the discretization. To control this source of errors we need first to estimate it, and then to be able to change the mesh when the error is too large.

Let us address the error coming from $(\mathbf{E 3})$. Let $\mathcal{A} \subset \mathbb{T}$ be the set of points corresponding to the table of values used in the algorithm. In the case of a Fourier representation of size $n_{\theta}$, then $\mathcal{A}=\left\{k / n_{\theta}\right\}_{k=0}^{n_{\theta}-1}$ is an equispaced mesh of [0,1). After some iterations of the Newton's Algorithm [5.4, we obtain a set of values $\left\{K\left(\theta_{i}\right)\right\}_{\theta_{i} \in \mathcal{A}}$ and an eccentricity $e$ satisfying the invariance equation in a mesh

$$
\max _{\theta_{i} \in \mathcal{A}}\left\|P_{e} \circ K\left(\theta_{i}\right)-K\left(\theta_{i}+\omega\right)\right\|<\delta,
$$

where $\delta$ is a fixed tolerance, e.g. $\approx 10^{-11}$ in double precision. Note that we are not fixing the norm in (31) which typically can be the sup-norm, the analytic norm, etc.

Let us now define $\delta^{*}$ as

$$
\delta^{*}=\max _{\theta \in \mathbb{T}}\left\|P_{e} \circ K(\theta)-K(\theta+\omega)\right\| .
$$

The computation of $\delta^{*}$ is in general difficult and we suggest two standard heuristic alternatives.

The first option is very fast: it consists in looking at the norm of some of the "last" Fourier coefficients and using it as an estimate for the truncation error of the series. Once the Newton's iteration has converged on a given mesh, we check the size of these coefficients. If one of them is larger than a prescribed threshold, we assume that the interpolation error is too big, and we increase the number of Fourier modes in the direction of these large coefficients.

The second option is to evaluate the error in (31) on a set of values $\widetilde{\mathcal{A}} \subset \mathbb{T}$ different from $\mathcal{A}$. One can use a thinner set $\widetilde{\mathcal{A}}$ to produce a better estimate of the invariance $\delta^{*}$. This procedure can be computationally expensive. An easier alternative is to consider $\tilde{\mathcal{A}}$ with the same number of points as $\mathcal{A}$. For instance,

$$
\widetilde{\mathcal{A}}=\mathcal{A}+v
$$

with $v$ equal to one half of the distance between points of $\mathcal{A}$ in the direction $\theta_{i} \in \mathcal{A}$. In the Fourier case, $\widetilde{\mathcal{A}}=\left\{(k+0.5) / n_{\theta}\right\}_{k=0}^{n_{\theta}-1}$ should be enough.

Hence, we have a new mesh $\widetilde{\mathcal{A}}$ which is interlaced with the initial mesh $\mathcal{A}$. Then, we check that

$$
\max _{\theta_{i} \in \widetilde{\mathcal{A}}}\left\|P_{e} \circ K\left(\theta_{i}\right)-K\left(\theta_{i}+\omega\right)\right\|<\delta .
$$

If this test is not satisfied, we add more Fourier coefficients and we go back to the Newton's iteration given by the Algorithm 5.4. If the test is satisfied, we can either stop and accept the solution or check it again with a thinner mesh. 
The key is then to avoid checking with thinner meshes during the computation as much as possible, because it is too costly, and to do just a single check at the end to ensure the accuracy.

§5.4. Implementation of the algorithm. Once Algorithm 5.4 is coded so that it becomes a sequence of arithmetic operations (and transcendental functions), it is almost easy to make it run in extended precision arithmetic, see Appendix D.

There are, however some caveats:

- One loses the hardware support.

- The hardware optimized libraries have to be replaced by hand coded libraries. Notably, one cannot use BLAS, LAPACK, or FFTW and they have to be substituted by explicit algorithms. We have used our own implementations.

- In iterative processes, one has to choose the stopping criteria appropriately. As standard, one writes the stopping criteria as a power of Machine epsilon.

- For us, the most important point is that, in order to achieve high accuracy of the ODE integration with a reasonable step in a reasonable amount of time, one needs a high order method.

We have used the Taylor's method, which is based on computing the Taylor's expansion of the solution of the equation to a very high order, see Appendix B.

The paper [JZ05a] presents a very general purpose generator of Taylor's integrators based on Automatic Differentiation. If one specifies (in a very simple format) a differential equation, the program taylor (supplied and documented in [JZ05a]) generates automatically an efficient Taylor solver written in C. The user can select whether this Taylor solver uses standard arithmetic or extended precision arithmetic (either GMP or MPFR). It is important that Taylor's methods can work well with different versions of the arithmetic.

One important product of the Taylor's integrator [GJJC $\left.{ }^{+} 21\right]$ is that we obtain very efficient solvers of the variational equations. We will report on them in Appendix C, since they are a natural extension of the Taylor's integrator. We note that we will not use them in the numerical experiments of this paper, but we will use them in CCGdlL20b. We remark that in mission design, they appear in the method of differential corrections.

§5.4.1. Using profilers to detect bottlenecks. The computation of invariant tori with the Newton method that we propose is remarkably fast when the initial guess is good enough. However, the continuation to the breakdown requires to increase the Fourier modes and then the computational cost will increase in proportion.

The key step in a continuation process is the correction of the solution for the new parameter values are being continued. In our case, it is the Newton step. We have used a $\mathrm{C}$ profiler in a single continuation step with multiprecision of 55 digits to realize which are the most CPU-time consuming parts. We did it for different values of $\varepsilon \in\left\{10^{-4}, 2 \cdot 10^{-4}\right\}$, $\eta \in\left\{10^{-3}, 10^{-6}\right\}, \omega \in\left\{\omega_{1}, \omega_{2}\right\}$, and $N \in\{128,256\}$ number of Fourier modes getting, in all of them, similar results. 
In average around $98.1 \%$ was dedicated to the Newton step correction, inside this step around $97.6 \%$ was for the evaluation of the ODE (and its variationals) in the ODE integrator and the correction of the integration stepsize. Inside of it, around $31 \%$ was for the addition of jet transport elements, $25 \%$ for multiplication, $18 \%$ for assignments, and $6.5 \%$ for scalar multiplications.

As consequence, we conclude that the FFT, the solvers of cohomological equations and the shiftings in Algorithm 5.4 are irrelevant in terms of CPU-time as well as the memory allocation. The second conclusion is that the ODE integration is the crucial part. This fact will be exploited and detailed in Section $\$ 5.5$.

§5.5. Parallelization. There are several operations in the Algorithm 5.4 that are fully independent to each other, such as those steps which are done in a table of values of $\theta$ and the solution of the cohomological equations using the Lemma 5.5.

The use of a profiler shows that the main bottleneck, in terms of CPU-time usage, is the ODE integration involved in $P_{e}$, given in (22), and its first order directional derivatives.

A simple concurrent parallelization for each of the different numerical integrations (previously ensuring that there is non-shared memory between the threads) shows an evident speed-up with respect to non-concurrent versions. In our case, we run the code with multiprecision arithmetic, in particular with MPFR, and we must be sure that each of the parallelized parts work correctly with the multiprecision. In the case ot MPFR we must initialize the precision and the rounding mode for each of the different CPU's.

Figure 3 shows the non-parallel execution times and the speed-ups of Algorithm 5.4 using the initial guess from Algorithm 5.6 with $\eta=10^{-6}, \varepsilon=10^{-4}, 135$ digits of precision and different number of modes in the Fourier representation. The figure was done in an Intel Xeon Gold $5220 \mathrm{CPU}$ at $2.20 \mathrm{GHz}$ with $18 \mathrm{CPUs}$ with hyperthreading which simulates 36 CPUs.

Note that the non-parallel data in Figure 3 is extremely well fit by

$$
T=0.430018 N+4.75918,
$$

where $T$ denotes the CPU time in seconds and $N$ the Fourier representation size. This means that the single core time scales behave (in practice) linearly with the size of the problem. The logarithmic correction appearing in the theory of the FFT does not seem to be observable which is due to the fact that the FFT is not the main problem in the performance of our method. Of course, this is significantly better than the $N^{3}$ of Newton's methods based on inverting matrices.

Algorithm 5.4 also accepts other concurrent computations such as the FFT algorithm or the solution of the cohomology equations in steps 8 and 12 . The latter did not show an important speed up, presumably because the time spent in these calculations is not so important overall (the number of points needed is not so large, due to our reduction to $1-\mathrm{D})$.

§5.6. A practical implementation of Algorithm 5.4. As an example of the implementation of Algorithm 5.4, we provide in Figure 4 the construction of the invariant attractors for (8) with a small dissipation, say $\eta=10^{-6}$, and frequencies given by (28) and (29). Figure 5 gives the results for a higher dissipation, namely $\eta=10^{-3}$. 


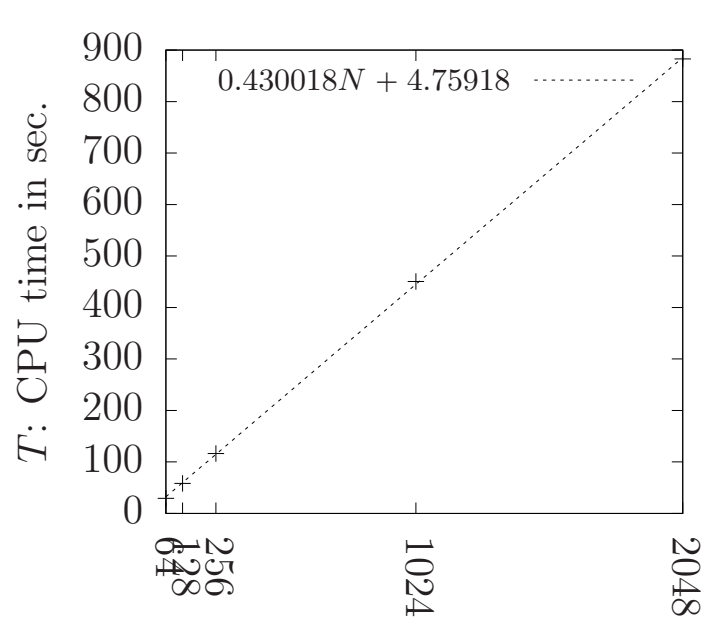

$N$ : Fourier size

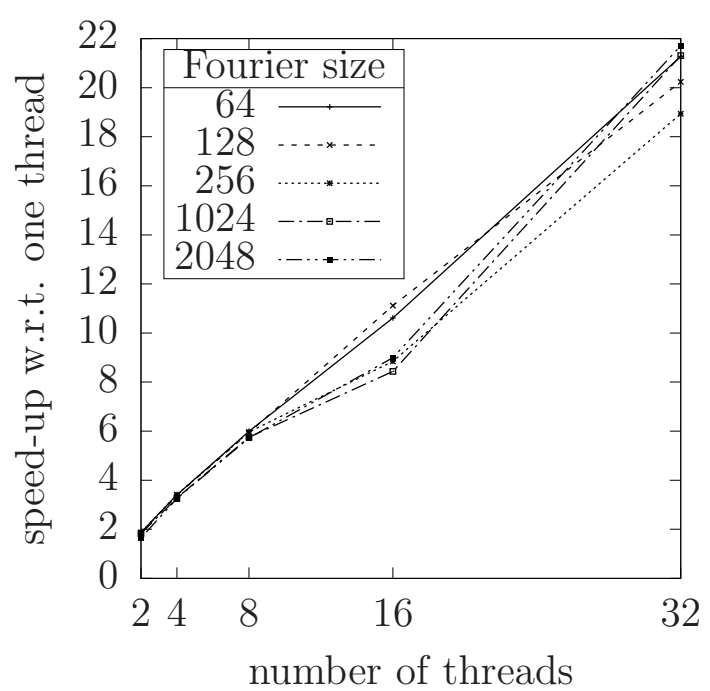

FiguRE 3. On the left: timings not using parallelization and fit of the times changing the number of Fourier modes. On the right: Speed-up by a parallel ODE integration in step 7 of Algorithm 5.4 for different number of threads and different number of Fourier modes.

Both figures show different invariant attractors when the perturbative parameter $\varepsilon$ changes by using a standard continuation procedure with interpolation. At each of these continuation steps with respect to $\varepsilon$ we apply Algorithm 5.4 with a tolerance $10^{-35}$, which refines the embedding $K_{\varepsilon}$ and the eccentricity $e_{\varepsilon}$, and we also check the accuracy, see Section $\$ 5.3$, to ensure that the numerical solution is accurate enough.

Additionally, we perform an extra refinement at each continuation step to ensure that the plots in Figures 4 and 5 make the value of $x$ in the range $[0,2 \pi)$. More precisely, if $\left(\tilde{K}_{\varepsilon}, e_{\varepsilon}\right)$ is the output of Algorithm [5.4, then we assign $K_{\varepsilon} \leftarrow \tilde{K}_{\varepsilon}$ with $K_{\varepsilon}^{1}(0)=0$. That is, we apply a shift $\alpha$ on $\tilde{K}_{\varepsilon}$ with $\alpha$ so that the first component of $\tilde{K}_{\varepsilon}$ at 0 is zero.

The results shown in Figure 4 give evidence of the effectiveness of the method of constructing invariant attractors, using the reduction to a map and the implementation of Algorithm 5.4.

\section{§5.7. An empirical comparison between the model with time dependent fric-} tion (8) and the average friction (10). In this section we present a comparison between the numerical results in the model with time-dependent dissipation (8) and the model with the averaged dissipation (10). In Appendix $₫$ we present two rigorous justifications of the averaging procedure.

In Figure 6, we plot the difference between the drift parameters (namely the eccentricities) of the full and averaged models for the tori with frequencies $\omega_{1}$ and $\omega_{2}$; such difference is small, say of the order of $10^{-7}$, even for parameter values close to breakdown. We also note that the $y$-axis in Figure 6 is just the difference (without absolute value) which means that $e>e_{\text {avg }}$ at each of the $\varepsilon$ values. 


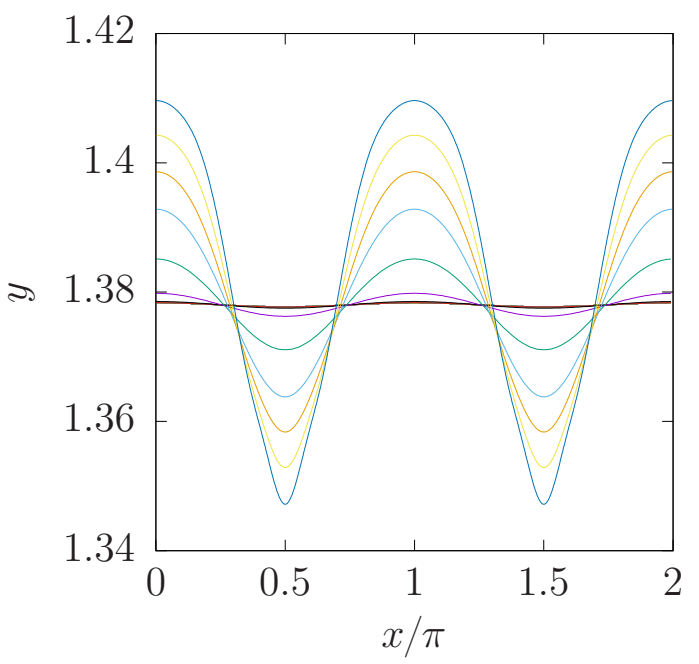

$\omega$ as in $(29)$ and $\eta=10^{-6}$
$\varepsilon=7.53113 \mathrm{E}-03 e=2.49466 \mathrm{E}-01$
$\varepsilon=9.53113 \mathrm{E}-03 \quad e=2.49043 \mathrm{E}-01$
$\varepsilon=1.15311 \mathrm{E}-02 \quad e=2.48553 \mathrm{E}-01$
$\varepsilon=1.22588 \mathrm{E}-02 \quad e=2.48363 \mathrm{E}-01$

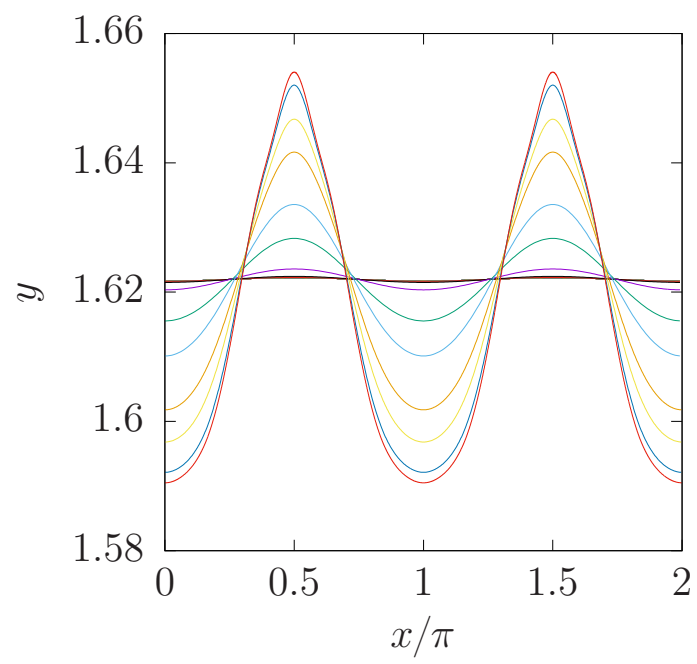

$\omega$ as in (28) and $\eta=10^{-6}$ $\varepsilon=4.93173 \mathrm{E}-03 e=3.15391 \mathrm{E}-01$ $\varepsilon=6.90324 \mathrm{E}-03 e=3.15698 \mathrm{E}-01$ $\varepsilon=8.90324 \mathrm{E}-03 e=3.16098 \mathrm{E}-01$ $\varepsilon=1.09032 \mathrm{E}-02 e=3.16569 \mathrm{E}-01$

FiguRE 4. Invariant attractor of (8) with a fixed dissipation $\eta=10^{-6}$ after a continuation w.r.t. the perturbative parameter $\varepsilon$. On the left, the frequency $\omega$ is like in (29) and on the right as in (28).

$\S 5.8$. Comment for double precision accuracy. The computation with multiprecision is already fast enough thanks to the quadratic convergence of our method. The use of multiprecision allows to get tori of arbitrary accuracy and, in particular, it helps to reach values of the parameter close to the breakdown. These calculations can be used to identify mathematical patterns close to breakdown and to use KAM theory. However, we can also perform double or even float precision computations for values that are not close to the breakdown. As one expects, the required time to converge using the Newton process presented in this paper, see Algorithm 5.4, is smaller than in multiprecision, paying the price of accuracy.

The first reason for this speed is because we request less accuracy -around $10^{-14}$ for double precision- which makes the algorithm converge in fewer iterations. The second reason is that we avoid the possible overhead of using a software package, such as MPFR, and we can then exploit the hardware optimizations provided by compilers.

After adapting our code to run with double precision we detect that the values of $\varepsilon$ for which our method still converges is around $7.8 \times 10^{-3}$ which is far from the $\varepsilon \sim 10^{-2}$ using more accuracy, the speed-up of the parallelization strategy, see $\S 5.5$. behaves similarly, and the time of a continuation step (with one CPU) decreases. It still depends on the number of Fourier modes and some example of values are reported in Table 1. 


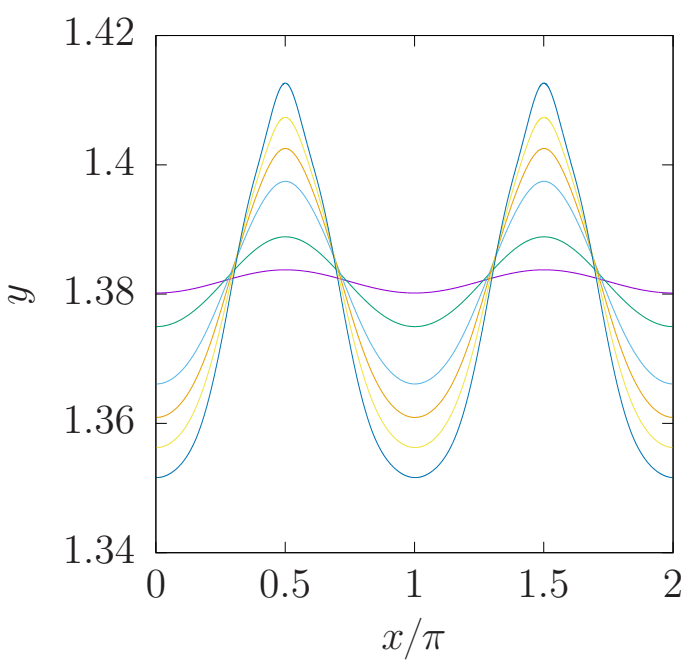

$$
\begin{aligned}
& \omega \text { as in }(29) \text { and } \eta=10^{-3} \\
& \varepsilon=5.87347 \mathrm{E}-03 \quad e=2.49751 \mathrm{E}-01 \\
& \varepsilon=7.87347 \mathrm{E}-03 \quad e=2.49400 \mathrm{E}-01 \\
& \varepsilon=9.75827 \mathrm{E}-03 \quad e=2.48991 \mathrm{E}-01 \\
& \varepsilon=1.17583 \mathrm{E}-02 \quad e=2.48494 \mathrm{E}-01
\end{aligned}
$$

FIGURE 5. Invariant attractor of (8) with a fixed dissipation $\eta=10^{-3}$ after a continuation w.r.t. the perturbative parameter $\varepsilon$. On the left, the frequency $\omega$ is like in (29) and on the right as in (28).

\begin{tabular}{c|cccccc}
$N$ & 64 & 128 & 256 & 512 & 1024 & 2048 \\
\hline$T$ & 0.570 & 1.132 & 2.280 & 4.618 & 9.329 & 18.049
\end{tabular}

TABLE 1. Example of time $T$ (in seconds) of a continuation step using double precision, $N$ Fourier modes, no parallelism, $\eta=10^{-3}, \omega$ as in (28), Newton's tolerance $10^{-14}$, and integration tolerance $10^{-16}$. The data fit extremely well with $T=0.00884 N+0.0561$.

In certain problems, it may be worth optimizing the speed (at the price of accuracy and programming time). In such cases where speed is the most important consideration, it may be worth taking advantage of modern computer architectures such as GPU's. (the very structured nature of our algorithm makes it a tantalizing possibility). We encourage these (or any other) developments on the methods presented here. 

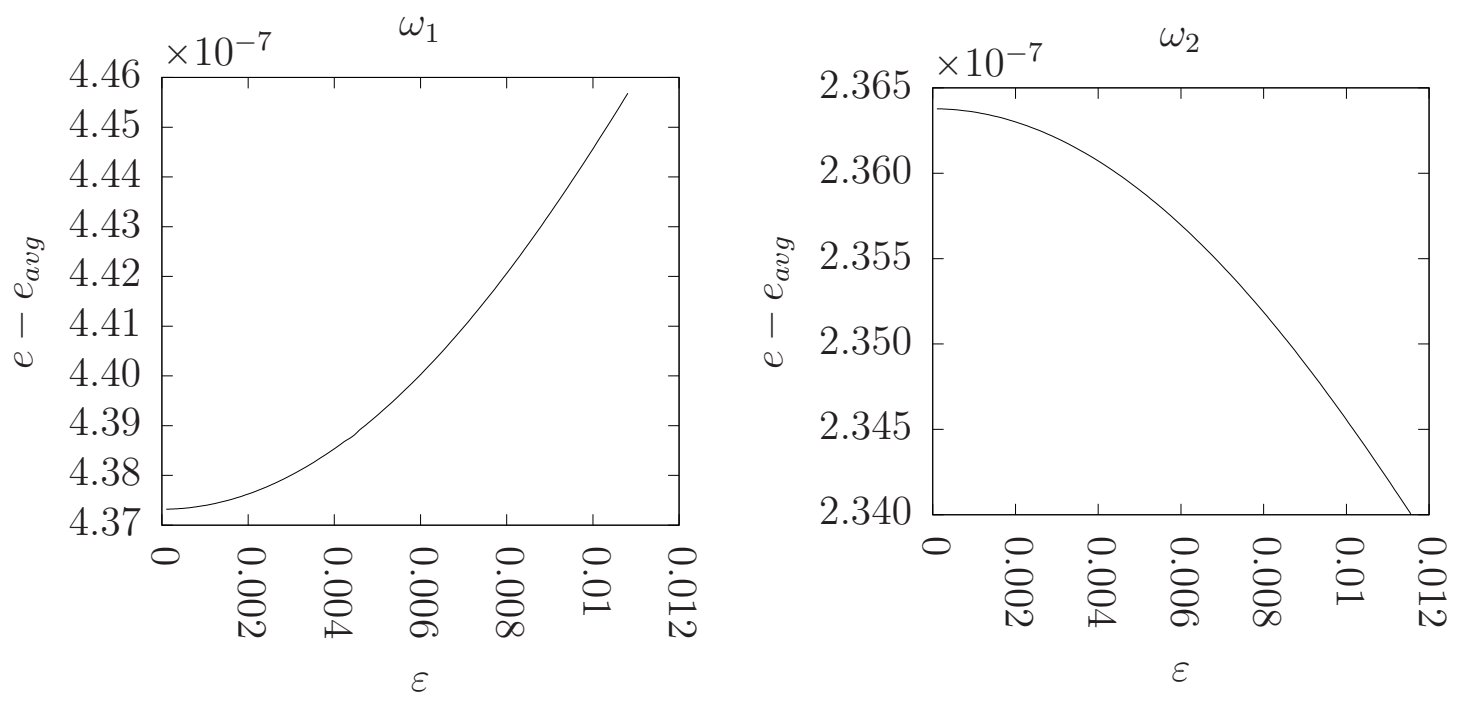

FiguRE 6. Difference of the eccentricities of the tori using the averaged (10) and the non-averaged (8) spin-orbit model in the case of dissipation $\eta=10^{-6}$ and for the two frequencies of interest $\omega_{1}$ in (28) on the left and $\omega_{2}$ in (29) on the right.

\section{§6. Conclusions}

There are several important features of the method developed in this paper for the construction of invariant tori using the return map; we highlight below some of these features.

- The method only requires dealing with functions of a low number of variables.

- The iterative step to construct the tori is quadratically convergent.

- The operation count and the operation requirements are low.

- The most costly step (integration of the equations) is very easy to parallelize efficiently. Many other steps of the algorithm involve vector operations, Fourier transforms, which also can take advantage of modern computers but here with less impact in the performance.

- The method is well adapted to the rather anisotropic regularity of KAM tori and, in the smooth direction, takes advantage of the developments in integration of ODE's.

- The method is reliable, since it is backed by rigorous a-posteriori theorems.

- The condition numbers required are evaluated in the approximate solution and do not involve global assumptions on the flow such as twist.

- From the theoretical point of view, the a-posteriori theorems give a justification of other heuristic methods that produce approximate solutions including asymptotic expansions and averaging methods. 


\section{Appendix A. Justification of AVERAging Methods}

In this section, we study the relation between the time dependent spin-orbit model (8) and the model (9) in which the dissipation is averaged.

Superficially, the relation between the two models and their attractors seems to be problematic even in the perturbative regime, since the existence of quasi-periodic solutions makes assertions for all the time and conventional averaging methods make assertions only for times which are inverse powers of the perturbation.

We will present two arguments showing that, indeed for the problem of the existence of quasi-periodic tori, the averaging method produces an accurate result. The first argument (Section A.1 will apply to very general models, but will produce results for KAM tori. The second method (Section A.2 will be very specific for the spin-orbit problem but will provide information for all orbits.

We note, however, that our method produces not only a rigorous estimate on the error of the averaging method, but also suggests other approximations that are more accurate than the usual procedure of averaging only the dissipation (see Section A.2). Our results show that, if besides averaging the dissipation, one changes slightly the conservative forces (we give explicit formulas), one gets results that are more accurate than just averaging the friction.

In this section we will consider a differential equation of the form

$$
\frac{d^{2} x(t)}{d t^{2}}+a(t) \frac{d x(t)}{d t}+F(x(t), t)=0
$$

In the perturbative case, we will have that there is a small parameter in the time dependence $a(t)=\mu \alpha(t)$.

A.1. Perturbative arguments based on a-posteriori theorems. We first observe that the key to our result is the study of the time-one map of the vector field.

If the return maps of two vector fields are close (in a smooth enough norm) and they satisfy the non-degeneracy conditions, the solutions of the invariance equation (26) for the two systems are close.

To detail this remark, let us consider two Poincaré maps $P_{e}^{(A)}$ and $P_{e}^{(C)}$, depending on a drift term $e$ and satisfying some non-degeneracy conditions; for example, we can take $P_{e}^{(A)}$ as the Poincaré map of the averaged system and $P_{e}^{(C)}$ as the Poincaré map of the complete, non-averaged system.

Let $\left(K_{A}, e_{A}\right)$ be an approximate solution of the invariance equation as in (27) with a small error term $E_{A}$ :

$$
P_{e_{A}}^{(A)} \circ K_{A}(\theta)-K_{A}(\theta+\omega)=E_{A}(\theta)
$$

Assume that the maps $P_{e}^{(C)}, P_{e}^{(A)}$ are close (in a suitable norm); then $\left(K_{A}, e_{A}\right)$ is an approximate solution for $P_{e}^{(C)}$; in fact, we have that

$$
\begin{aligned}
P_{e_{A}}^{(C)} \circ K_{A}(\theta)-K_{A}(\theta+\omega) & =\left(P_{e_{A}}^{(C)}-P_{e_{A}}^{(A)}\right) \circ K_{A}(\theta)+P_{e_{A}}^{(A)} \circ K_{A}(\theta)-K_{A}(\theta+\omega) \\
& =\left(P_{e_{A}}^{(C)}-P_{e_{A}}^{(A)}\right) \circ K_{A}(\theta)+E_{A}(\theta) \equiv E_{C}(\theta)
\end{aligned}
$$


with $E_{C}$ small. Using the KAM theory given in CCdlL13a, there exists $\left(K_{C}, e_{C}\right)$ such that

$$
P_{e_{C}}^{(C)} \circ K_{C}(\theta)-K_{C}(\theta+\omega)=0 ;
$$

besides, the results in CCdlL13a show that $K_{C}$ is close to $K_{A}$ and $e_{C}$ is close to $e_{A}$.

The importance of this remark is that to obtain the distance of the maps, we only need to estimate the difference between the evolution for a time $2 \pi$. In the perturbative regime, when the oscillation is of size $\mu$ controlling the distance between the solution of the averaged and non-averaged systems is well within the reach of standard averaging methods [Hal80, SVM07] (which deal well with times $O\left(\mu^{-1}\right)$, much larger than $2 \pi$ ).

\section{A.2. Non-perturbative arguments based on elementary scaling of variables}

and of time. In this section, we present a completely elementary (non perturbative) technique to justify the averaging method.

We just observe that if $x$ satisfes (33) then, for any non-zero (smooth) function $\gamma(t)$, the function $y(t)$ defined by $x(t)=\gamma(t) y(t)$ satisfies:

$$
\gamma(t) \frac{d^{2} y(t)}{d t^{2}}+\left(2 \gamma^{\prime}(t)+a(t) \gamma(t)\right) \frac{d y(t)}{d t}+\left(\gamma^{\prime \prime}(t)+a(t) \gamma^{\prime}(t)\right) y(t)+F(\gamma(t) y(t), t)=0
$$

Let $\bar{a}$ be the average value of $a(t)$; if we choose

$$
\gamma(t)=\exp \left(-\frac{1}{2} \int_{0}^{t}(a(s)-\bar{a}) d s\right),
$$

which is a periodic function, the equation (34) becomes:

$$
\frac{d^{2} y(t)}{d t^{2}}+\bar{a} \frac{d y(t)}{d t}+G(y(t), t)=0
$$

where

$$
G(y, t):=\frac{\gamma^{\prime \prime}(t)+a(t) \gamma^{\prime}(t)}{\gamma(t)} y+\frac{1}{\gamma(t)} F(\gamma(t) y, t)
$$

Hence, the function $y$ satisfies the equation with an average dissipation (and a different $F)$. Notice that $\gamma(0)=\gamma(2 \pi)=1$, so that

$$
\begin{aligned}
x(0) & =y(0) \gamma(0) \\
x(2 \pi) & =y(2 \pi) \gamma(2 \pi) \\
y^{\prime}(0) & =x^{\prime}(0)+\frac{1}{2}(a(0)-\bar{a}) x(0) \\
y^{\prime}(2 \pi) & =x^{\prime}(2 \pi)+\frac{1}{2}(a(2 \pi)-\bar{a}) x(2 \pi) .
\end{aligned}
$$

We can think of (38) as a change of variables in phase space from $\left(x, x^{\prime}\right)$ to $\left(y, y^{\prime}\right)$. The return map for the averaged equation (36) in the variables $\left(y, y^{\prime}\right)$ is equivalent to the orginal problem.

Hence, we can read off the original return map as the return map of the averaged equation under a change of variables. 
Note that, as standard in the averaging method, the relation between the averaged equations and the true ones is mainly a change of variables and a modification to the equations. Note, that in our very simple equations, the equivalence is exact.

In the perturbative case, we obtain that $\gamma=1+O(\varepsilon)$ and that the function $G$ in (37) satisfies $G-F=O(\varepsilon)$; also the change of variables between $\left(x, x^{\prime}\right)$ and $\left(y, y^{\prime}\right)$ is $O(\varepsilon)$ close to the identity.

Our treatment shows that models with average dissipation should involve also a change of variables and a modification of the forces.

\section{ApPEndix B. TAYLOR'S INTEGRATION METHOdS}

Taylor's method is one of the most common numerical integration techniques of an initial value problem of an ordinary differential equation of the form

$$
\begin{aligned}
\dot{z} & =F(z, t), \\
z\left(t_{0}\right) & =z_{0} .
\end{aligned}
$$

The Taylor's method is competitive, in speed and accuracy, with respect to other standards methods. The main drawback is that the Taylor's method is an explicit method, so it has all limitations of these kind of schemes, for example it is non-appropriate for stiff systems.

The idea behind the Taylor's method is very simple. Given the initial condition $z\left(t_{0}\right)=$ $z_{0}$, the value $z\left(t_{1}\right)$, with $t_{1}=t_{0}+h$, is approximated by the Taylor series of $z(t)$ at $t=t_{0}$. The Taylor series is truncated up to an order, say $N$, to try to ensure the absolute/relative tolerances requested during the numerical integration. Therefore, to get the solution $z_{1}$ at time $t=t_{1}$ from the solution $z_{0}$ at time $t=t_{0}$ we consider the expression

$$
z_{1}=z_{1}+z_{0}^{[1]} h+z_{0}^{[2]} h^{2}+\cdots+z_{0}^{[N]} h^{N}
$$

where $z_{0}^{[k]}, k=1, \ldots, N$, represents the normalized derivative at order $k$ computed at $t_{0}$, i.e.,

$$
z_{0}^{[k]}=\frac{1}{k !} \frac{d^{k} z}{d t^{k}}\left(t_{0}\right)
$$

Using the coefficients of the Taylor's expansion in (40) one can estimate the range of $h$ where the Taylor series is valid (up to a tolerance). This fact makes the Taylor's method suitable for multi-precision arithmetic.

The computation of the derivatives might be a difficult task, which can be lightened by using automatic differentiation (see, for instance, [RC96, GW08]), thus providing very efficient implementations of Taylor's method as illustrated in JZ05b] to which we refer for full details. We recall that automatic differentiation provides a recursive computation of operations on polynomials, which implies the manipulation of formal power series.

We also mention that jet transport (see Appendix $\mathrm{E}$ ), namely automatic differentiation with respect to initial data and parameters, can be used in Taylor's method to approximate the high order variational flow as it has been proved in [GJJC ${ }^{+21}$. 


\section{Appendix C. VARIATIONAL EQUATIONS FOR THE SPIN-ORBIT PROBLEM}

In this section we provide the formulae for the computation of the variational equations with respect to coordinates and parameters, motivated by the fact that - even if not used in the present paper - they are useful in different contexts, like the parameterization of invariant objects, estimates based on derivatives of the flow, computation of chaos indicators.

C.1. Variational equations. The variation with respect to the initial conditions of (15) involves the Jacobian whose elements are given by

$$
\begin{aligned}
& a_{11}=0, \quad a_{21}=-2 \varepsilon \frac{a}{r(u ; e)} c(\beta ; u, e), \\
& a_{12}=1, \quad a_{22}=e \frac{a}{r(u ; e)} \sin u-\eta\left(\frac{a}{r(u ; e)}\right)^{5}
\end{aligned}
$$

with $c(\beta ; u, e)$ defined in (14).

The variations with respect to the initial conditions for the system (15) or for the system (8) are not the same, although some properties such as the determinant or the eigenvalues of the $2 \pi$-time map are preserved. The explicit relation between the two variations is given by

$$
\begin{aligned}
\frac{\partial x}{\partial x_{0}}(t) & =\frac{\partial \beta}{\partial \beta_{0}}(u), & \frac{\partial x}{\partial y_{0}}(t) & =\frac{\partial \beta}{\partial \gamma_{0}}(u)\left(1-e \cos u_{0}\right), \\
\frac{\partial y}{\partial x_{0}}(t) & =\frac{\partial \gamma}{\partial \beta_{0}}(u) \frac{1}{1-e \cos u}, & \frac{\partial y}{\partial y_{0}}(t) & =\frac{\partial \gamma}{\partial \gamma_{0}}(u) \frac{1-e \cos u_{0}}{1-e \cos u},
\end{aligned}
$$

where $\left(x_{0}, y_{0}\right)=\left(\beta_{0}, \gamma_{0} /\left(1-e \cos u_{0}\right)\right)$. The relation (42) must be interpreted as follows: after the integration of (15) and its first variational equations, which uses the terms in (41), from time $u_{0}$ to $u$ and with initial condition $\left(\beta_{0}, \gamma_{0}\right) \in[0,2 \pi) \times \mathbb{R}$, then the variation with respect to the initial condition $\left(x_{0}, y_{0}\right)$ in (8) from the initial time $t_{0}=u_{0}-e \sin u_{0}$ to the final time $t=u-e \sin u$ is given by the relations in (42).

Note that (42) is simplified when $u_{0}=0$ and $u=2 \pi$.

C.2. Variational equations with respect to the parameters. The variational equations with respect to the parameters $\varepsilon$ and $\eta$ of the system (15) are quite straightforward as well as their relations in terms of the variables $(x, y)$. They are given by the following expressions:

$$
\frac{\partial x}{\partial \star}(t)=\frac{\partial \beta}{\partial \star}(u) \text { and } \frac{\partial y}{\partial \star}(t)=\frac{\partial \gamma}{\partial \star}(u) \frac{a}{r}, \quad \star \in\{\varepsilon, \eta\}
$$

However, the case for the parameter $e$ in (15) requires a little bit more of work and its relation with respect to the coordinates $(x, y)$ also involves more terms which we make 
explicit below:

$$
\begin{aligned}
\frac{\partial x}{\partial e}(t)= & \frac{\partial \beta}{\partial e}(u)-\frac{\partial \beta}{\partial \gamma_{0}}(u) \frac{\gamma_{0} \cos u_{0}}{1-e \cos u_{0}}+\gamma(u) \frac{a}{r} \sin u \\
& +\left[\frac{\partial \beta}{\partial u_{0}}(u)+\frac{\partial \beta}{\partial \gamma_{0}}(u) \frac{\gamma_{0} e \sin u_{0}}{1-e \cos u_{0}}\right] \frac{\sin u_{0}}{1-e \cos u_{0}} \\
\frac{\partial y}{\partial e}(t)= & \frac{\partial \gamma}{\partial e}(u) \frac{a}{r}+\gamma(u)\left(\frac{a}{r}\right)^{2} \cos u-\frac{\partial \gamma}{\partial \gamma_{0}}(u) \frac{a}{r} \frac{\gamma_{0} \cos u_{0}}{1-e \cos u_{0}} \\
& +\left[\frac{\partial \gamma}{\partial u}(u)-\gamma(u) \frac{a}{r} e \sin u\right]\left(\frac{a}{r}\right)^{2} \sin u \\
& +\left[\frac{\partial \gamma}{\partial u_{0}}(u)+\frac{\partial \gamma}{\partial \gamma_{0}}(u) \frac{a}{r} \frac{\gamma_{0} e \sin u_{0}}{1-e \cos u_{0}}\right] \frac{\sin u_{0}}{1-e \cos u_{0}} .
\end{aligned}
$$

Note that (44) is simplified when $u_{0}=0$ and $u=2 \pi$.

C.3. High order variational equations. In many cases the first order variational equations are straightforward and one can explicitly write them down in the numerical integrator. However, high order variational equations are cumbersome and the use of jet transport becomes highly recommended, see [GJJC ${ }^{+} 21$. The jet transport is also useful in the study of other structures such as stable manifolds (which will not be considered here).

Jet transport uses automatic differentiation (see GW08]), which manipulates multivariate polynomials to carry out the truncated Taylor's approximation containing (in the case of jet transport) the higher order variational flow, see [GJJC ${ }^{+} 21$ for a precise formulation. We also refer to Appendix $\mathrm{B}$ for a discussion of a polynomial manipulator up to degree 2 .

In the case of the spin-orbit problem given by (15), the polynomial manipulator must at least contain the sum, product, sine, cosine, and power operations. All of them have explicit recurrence expressions $\left(\left[\mathrm{Knu} 97, \mathrm{HCF}^{+} 16\right]\right)$. By the use of the polynomial manipulator, expressions such as (44) for higher orders are automatically obtained.

\section{Appendix D. Different models of COMputer ARithmetiC}

The Algorithm 5.4 can be implemented with multi precision arithmetic. The idea is that there are different models of computer arithmetic implementation. It is very easy to switch between different models using features of modern languages such as overloading.

We recall that computers deal only with representable numbers, which are just a (finite) subset of the real numbers and whose elements have the form

$$
\pm m \cdot \beta^{e-t}
$$

where $m \in\left[0, \beta^{t}-1\right]$ is an integer called mantissa, $\beta$ is the base or radix (typically $\beta=2$ ), $t$ is a positive integer denoting the precision and $s \in\left[s_{\min }, s_{\max }\right]$ is also an integer called 
the exponent. Typical values in the double precision arithmetic following the IEEE 754 standard 2 are $\beta=2, t=53, s_{\min }=-1021$, and $s_{\max }=1024$.

Performing arithmetic operations on representable numbers, very often yields numbers that are not representable. Sometimes, the results are in the middle of representable numbers and then, one assigns the result to one of the neighboring numbers. This is called rounding. There are several rules that are in common use: rounding to nearest, rounding down, rounding up, rounding towards zero, rounding away to zero. There are operations that yield a number that is far from any representable number (e.g., adding the largest representable number to itself). One usually represents those as Inf. Of course, one needs to have rules on how to deal with Inf (e.g., does one distinguish between positive/negative infinity). Finally, there operations that do not make sense, such as division by zero.

In the IEEE 754 standard, it is specified that there is a so called control word. The bits of the control word specify the rounding modes and how to treat infinity.

Finally, we just note that, when the result of two numbers is very close to zero, rounding leads to a great loss of precision. IEEE 754 has introduced also the denormalized numbers which make the gaps near zero smaller, even if they require specialized rules to be handled.

We also note that the IEEE 754 standard specifies the calculations of some transcendental functions subject to the same rules of rounding.

Nowadays, the IEEE 754 standard is implemented in hardware. Both in CPU's and in many GPU's. Many important libraries (BLAS/ATLAS, FFTW3) take advantage of the availability of hardware and obtain advantages in speed. Having very reliable rounding modes that satisfy identities allows to improve also the accuracy. For example, there are algorithms that sum a sequence of numbers with a roundoff error independent of the length of the sequence to be summed. One can also use interval arithmetic that provides rigorous estimates of the arithmetic operations.

Nowadays, there exist libraries that provide the same capabilities indicated above (representable numbers of the standard form, rounding modes, etc.), but with a number of digits that can be selected at run time; for example, MPFR [FHL ${ }^{+}$07] (which is the one we have used) or some other.

Using modern programming techniques such as overloading, it is not difficult to write versions of our programs for the arithmetic using the hardware or in MPFR and select the precision.

It is important to remark that in a parallel scenario, one must be sure that the global variables used in these libraries are initialized in each of the different threads. In particular, in the MPFR case, one needs to initialize the precision and the rounding for each of the threads, otherwise the output will differ from the non-parallel version.

\section{Appendix E. A goodness test FOR JET TRAnsport}

Let $\dot{x}=f(t, x)$ an ODE in $\mathbb{R}^{d}$ with flow denoted by $\varphi(t ; x)$. The test consists in running three integrations with two different integrators.

\footnotetext{
${ }^{2}$ We omit the discussion of "denormalized numbers", perhaps the aspect of IEEE 754 that generated the most controversy.
} 
First, we run $x_{0}+s$ with an integrator with jet transport of order, say $N$, up to a time, say 1 . The output is the jet $y(s)$ of order $N$.

Second, we choose a unitary vector $v$ and a scalar $h$ (typically small, say $h=10^{-7}$ ) and we define the quantity

$$
c_{h}=\left\|y(h)-\varphi\left(1 ; x_{0}+h v\right)\right\|,
$$

which is expected to behave as $c_{h} \approx c h^{N+1}$ for some $c>0$.

The third and last run is to repeat the second one, but now with $h / 2$ to get the quantity $c_{h / 2}$.

Finally, it must happen that

$$
\frac{c_{h}}{c_{h / 2}} \approx 2^{N+1}
$$

Therefore, the test is successful when $\log _{2}\left(c_{h} / c_{h / 2}\right) \approx N+1$, being $N$ the order of jets in the first integration.

Notice that (46) may suffer loss of precision if $h$ is too small. Therefore, one needs to choose a suitable $h$ by systematically trying several choices.

\section{REFERENCES}

[AKdlL07] Raymond A. Adomaitis, Ioannis G. Kevrekidis, and Rafael de la Llave. A computerassisted study of global dynamic transitions for a noninvertible system. Internat. J. Bifur. Chaos Appl. Sci. Engrg., 17(4):1305-1321, 2007.

[ALM00] Lluís Alsedà, Jaume Llibre, and MichałMisiurewicz. Combinatorial dynamics and entropy in dimension one, volume 5 of Advanced Series in Nonlinear Dynamics. World Scientific Publishing Co., Inc., River Edge, NJ, second edition, 2000.

[Arn63] V. I. Arnol'd. Proof of a theorem of A. N. Kolmogorov on the invariance of quasi-periodic motions under small perturbations. Russian Math. Surveys, 18(5):9-36, 1963.

[Ath98] Konstantin Athanassopoulos. Rotation numbers and isometries. Geom. Dedicata, 72(1):113, 1998.

[Ban02] A. Banyaga. Some properties of locally conformal symplectic structures. Comment. Math. Helv., 77(2):383-398, 2002.

[Bel01] V. V. Beletsky. Essays on the motion of celestial bodies. Birkhäuser Verlag, Basel, 2001. Translated from the Russian by Andrei Iacob.

[BM98] M. Berz and K. Makino. Verified integration of ODEs and flows using differential algebraic methods on high-order Taylor models. Reliab. Comput., 4(4):361-369, 1998.

[CC09] Alessandra Celletti and Luigi Chierchia. Quasi-periodic attractors in celestial mechanics. Arch. Ration. Mech. Anal., 191(2):311-345, 2009.

[CC10] R. Calleja and A. Celletti. Breakdown of invariant attractors for the dissipative standard map. CHAOS, 20(1):013121, 2010.

[CCdlL13a] R. Calleja, A. Celletti, and R. de la Llave. A KAM theory for conformally symplectic systems: efficient algorithms and their validation. J. Differential Equations, 255(5):9781049, 2013.

[CCdlL13b] Renato C. Calleja, Alessandra Celletti, and Rafael de la Llave. A KAM theory for conformally symplectic systems: efficient algorithms and their validation. J. Differential Equations, 255(5):978-1049, 2013.

[CCdlL20] Renato C. Calleja, Alessandra Celletti, and Rafael de la Llave. Kam estimates for the dissipative standard map. 2020.

[CCGdlL20a] Renato C. Calleja, Alessandra Celletti, Joan Gimeno, and Rafael de la Llave. Breakdown threshold of invariant attractors in the dissipative spin-orbit problem. Preprint, 2020. 
[CCGdlL20b] Renato C. Calleja, Alessandra Celletti, Joan Gimeno, and Rafael de la Llave. KAM estimates in the dissipative spin-orbit problem. Preprint, 2020.

[CdlL10] R. Calleja and R. de la Llave. A numerically accessible criterion for the breakdown of quasi-periodic solutions and its rigorous justification. Nonlinearity, 23(9):2029-2058, 2010.

[Cel90a] Alessandra Celletti. Analysis of resonances in the spin-orbit problem in celestial mechanics: higher order resonances and some numerical experiments. II. Z. Angew. Math. Phys., 41(4):453-479, 1990.

[Cel90b] Alessandra Celletti. Analysis of resonances in the spin-orbit problem in celestial mechanics: the synchronous resonance. I. Z. Angew. Math. Phys., 41(2):174-204, 1990.

[Cel10] Alessandra Celletti. Stability and Chaos in Celestial Mechanics. Springer-Verlag, Berlin; published in association with Praxis Publishing, Chichester, 2010.

[CF12] R. Calleja and J.-L. Figueras. Collision of invariant bundles of quasi-periodic attractors in the dissipative standard map. Chaos, 22(3):033114, 10, 2012.

[CL04] Alexandre C. M. Correia and Jacques Laskar. Mercury's capture into the $3 / 2$ spin-orbit resonance as a result of its chaotic dynamics. Nature, 429(6994):848-850, June 2004.

[CL14] A. Celletti and C. Lhotka. Transient times, resonances and drifts of attractors in dissipative rotational dynamics. Commun. Nonlinear Sci. Numer. Simul., 19(9):3399-3411, 2014.

[CPS15] A. Celletti, G. Pucacco, and D. Stella. Lissajous and halo orbits in the restricted threebody problem. J. Nonlinear Sci., 25(2):343-370, 2015.

[DSSY17] S. Das, Y. Saiki, E. Sander, and J.A. Yorke. Quantitative quasiperiodicity. Nonlinearity, 30(11):4111-4140, 2017.

$\left[\mathrm{FHL}^{+} 07\right] \quad$ L. Fousse, G. Hanrot, V. Lefèvre, P. Pélissier, and P. Zimmermann. MPFR: a multipleprecision binary floating-point library with correct rounding. ACM Trans. Math. Software, 33(2):Art. 13, 15, 2007.https://www.mpfr.org.

[FLG97] Claude Froeschlé, Elena Lega, and Robert Gonczi. Fast Lyapunov indicators. Application to asteroidal motion. Celestial Mech. Dynam. Astronom., 67(1):41-62, 1997.

[GJJC $\left.{ }^{+} 21\right] \quad J$. Gimeno, À. Jorba, M. Jorba-Cuscó, N. Miguel, and M. Zou. Numerical integration of high order variational equations of odes. 2021.

[GM01] G. Gómez and J. M. Mondelo. The dynamics around the collinear equilibrium points of the RTBP. Phys. D, 157(4):283-321, 2001.

[GMS10a] Gerard Gómez, Josep-Maria Mondelo, and Carles Simó. A collocation method for the numerical Fourier analysis of quasi-periodic functions. I. Numerical tests and examples. Discrete Contin. Dyn. Syst. Ser. B, 14(1):41-74, 2010.

[GMS10b] Gerard Gómez, Josep-Maria Mondelo, and Carles Simó. A collocation method for the numerical Fourier analysis of quasi-periodic functions. II. Analytical error estimates. Discrete Contin. Dyn. Syst. Ser. B, 14(1):75-109, 2010.

[GW08] A. Griewank and A. Walther. Evaluating derivatives. Society for Industrial and Applied Mathematics (SIAM), Philadelphia, PA, second edition, 2008. Principles and techniques of algorithmic differentiation.

[Hal75] Ole H. Hald. On a Newton-Moser type method. Numer. Math., 23:411-426, 1975.

[Hal80] Jack K. Hale. Ordinary differential equations. Robert E. Krieger Publishing Co., Inc., Huntington, N.Y., second edition, 1980.

$\left[\mathrm{HCF}^{+} 16\right]$ Àlex Haro, Marta Canadell, Jordi-Lluís Figueras, Alejandro Luque, and Josep-Maria Mondelo. The parameterization method for invariant manifolds, volume 195 of Applied Mathematical Sciences. Springer, [Cham], 2016. From rigorous results to effective computations.

[HdlL] A. Haro and R. de la Llave. Spectral theory and dynamical systems.

[HdlL06] À. Haro and R. de la Llave. A parameterization method for the computation of invariant tori and their whiskers in quasi-periodic maps: numerical algorithms. Discrete Contin. Dyn. Syst. Ser. B, 6(6):1261-1300 (electronic), 2006. 
[HM21] A. Haro and J. M. Mondelo. Flow map parameterization methods for invariant tori in hamiltonian systems. 2021.

[HNW93] E. Hairer, S. P. Nørsett, and G. Wanner. Solving ordinary differential equations. I, volume 8 of Springer Series in Computational Mathematics. Springer-Verlag, Berlin, second edition, 1993. Nonstiff problems.

[JM99] À. Jorba and J. Masdemont. Nonlinear dynamics in an extended neighbourhood of the translunar equilibrium point. In Hamiltonian systems with three or more degrees of freedom (S'Agaró, 1995), volume 533 of NATO Adv. Sci. Inst. Ser. C Math. Phys. Sci., pages 430434. Kluwer Acad. Publ., Dordrecht, 1999.

[JO09] Àngel Jorba and Estrella Olmedo. On the computation of reducible invariant tori on a parallel computer. SIAM J. Appl. Dyn. Syst., 8(4):1382-1404, 2009.

[JZ05a] À. Jorba and M. Zou. A software package for the numerical integration of ODEs by means of high-order Taylor methods. Experiment. Math., 14(1):99-117, 2005.

[JZ05b] Àngel Jorba and Maorong Zou. A software package for the numerical integration of ODEs by means of high-order Taylor methods. Experiment. Math., 14(1):99-117, 2005.

[KAdlL21a] Bhanu Kumar, Rodney L. Anderson, and Rafael de la Llave. Rapid and accurate computation of whiskered tori and their manifolds near resonances in periodically perturbed planar circular restricted 3-body problems. 2021.

[KAdlL21b] Bhanu Kumar, Rodney L. Anderson, and Rafael de la Llave. Using GPU's and the parameterization method for rapid search and refinement of connections between tori in periodically perturbed planar circular restricted 3-body problem. (AAS-349), 2021.

[Knu97] Donald E. Knuth. The art of computer programming. Vol. 2: Seminumerical algorithms. Addison-Wesley Publishing Co., Reading, Mass.-London-Don Mills, Ont, third revised edition, 1997.

[Kol54] A. N. Kolmogorov. On conservation of conditionally periodic motions for a small change in Hamilton's function. Dokl. Akad. Nauk SSSR (N.S.), 98:527-530, 1954. English translation in Stochastic Behavior in Classical and Quantum Hamiltonian Systems (Volta Memorial Conf., Como, 1977), Lecture Notes in Phys., 93, pages 51-56. Springer, Berlin, 1979.

[Las99] Jacques Laskar. Introduction to frequency map analysis. In Hamiltonian systems with three or more degrees of freedom (S'Agaró, 1995), volume 533 of NATO Adv. Sci. Inst. Ser. C Math. Phys. Sci., pages 134-150. Kluwer Acad. Publ., Dordrecht, 1999.

[LFC92] Jacques Laskar, Claude Froeschlé, and Alessandra Celletti. The measure of chaos by the numerical analysis of the fundamental frequencies. Application to the standard mapping. Phys. D, 56(2-3):253-269, 1992.

[Mac64] Gordon J. F. Macdonald. Tidal Friction. Reviews of Geophysics and Space Physics, 2:467541, January 1964.

[Mat68] John N. Mather. Characterization of Anosov diffeomorphisms. Nederl. Akad. Wetensch. Proc. Ser. A 71 = Indag. Math., 30:479-483, 1968.

[Mos62] J. Moser. On invariant curves of area-preserving mappings of an annulus. Nachr. Akad. Wiss. Göttingen Math.-Phys. Kl. II, 1962:1-20, 1962.

[Mos73] J. Moser. Stable and Random Motions in Dynamical Systems. Princeton University Press, Princeton, N. J., 1973.

[Oli16] Zubin P. Olikara. Computation of quasi-periodic tori and heteroclinic connections in astrodynamics using collocation techniques. ProQuest LLC, Ann Arbor, MI, 2016. Thesis (Ph.D.)-University of Colorado at Boulder.

[Pea05] S. J. Peale. The free precession and libration of Mercury. Icarus, 178(1):4-18, November 2005.

[RC96] Louis B. Rall and George F. Corliss. An introduction to automatic differentiation. In Computational differentiation (Santa Fe, NM, 1996), pages 1-18. SIAM, Philadelphia, PA, 1996. 
[SL12] Letizia Stefanelli and Ugo Locatelli. Kolmogorov's normal form for equations of motion with dissipative effects. Discrete Contin. Dynam. Systems, 17(7):2561-2593, 2012.

[SL15] Letizia Stefanelli and Ugo Locatelli. Quasi-periodic motions in a special class of dynamical equations with dissipative effects: A pair of detection methods. Discrete Contin. Dyn. Syst. Ser. B, 20(4):1155-1187, 2015.

[SNS10] J. Sánchez, M. Net, and C. Simó. Computation of invariant tori by Newton-Krylov methods in large-scale dissipative systems. Phys. D, 239(3-4):123-133, 2010.

[SV06] Tere M. Seara and Jordi Villanueva. On the numerical computation of Diophantine rotation numbers of analytic circle maps. Phys. D, 217(2):107-120, 2006.

[SVM07] J. A. Sanders, F. Verhulst, and J. Murdock. Averaging methods in nonlinear dynamical systems, volume 59 of Applied Mathematical Sciences. Springer, New York, second edition, 2007.

[WPM84] J. Wisdom, S. J. Peale, and F. Mignard. The chaotic rotation of Hyperion. Icarus, 58(2):137-152, May 1984.

Department of Mathematics and Mechanics, immas, National Autonomous University of Mexico (UnAM), App. Postal 20-726, C.P. 0100, Mexico D.F. (Mexico)

Email address: celleja@mym.iimas.unam.mx

Department of Mathematics, University of Rome Tor Vergata, Via della Ricerca Scientifica 1, 00133 Rome (Italy)

Email address: celletti@mat.uniroma2.it

Department of Mathematics, University of Rome Tor Vergata, Via della Ricerca Scientifica 1, 00133 Rome (Italy)

Email address: gimeno@mat.uniroma2.it

School of Mathematics, Georgia Institute of Technology, 686 Cherry St., Atlanta GA. 30332-0160 (USA)

Email address: rafael.delallave@math.gatech.edu 